\title{
Similarity Theory in the Surface Layer of Large-Eddy Simulations of the Wind-, Wave-, and Buoyancy-Forced Southern Ocean
}

\author{
William G. Large, Edward G. Patton, Alice K. DuVivier, And Peter P. Sullivan \\ National Center for Atmospheric Research, Boulder, Colorado \\ LEONEL ROMERO \\ University of California, Santa Barbara, Santa Barbara, California
}

(Manuscript received 22 August 2018, in final form 13 June 2019)

\begin{abstract}
Monin-Obukhov similarity theory is applied to the surface layer of large-eddy simulations (LES) of deep Southern Ocean boundary layers. Observations from the Southern Ocean Flux Station provide a wide range of wind, buoyancy, and wave (Stokes drift) forcing. Two No-Stokes LES are used to determine the extent of the ocean surface layer and to adapt the nondimensional momentum and buoyancy gradients, as functions of the stability parameter. Stokes-forced LES are used to modify this parameter for wave effects, then to formulate dependencies of Stokes similarity functions on a Stokes parameter $\xi$. To account for wind-wave misalignment, the dimensional analysis is extended with two independent variables, namely, the production of turbulent kinetic energy in the surface layer due to Stokes shear and the total production, so that their ratio gives $\xi$. Stokes forcing is shown to reduce vertical shear more than stratification, and to enhance viscosity and diffusivity by factors up to 5.8 and 4.0, respectively, such that the Prandtl number can exceed unity. A practical parameterization is developed for $\xi$ in terms of the meteorological forcing plus a Stokes drift profile, so that the Stokes and stability similarity functions can be combined to give turbulent velocity scales. These scales for both viscosity and diffusivity are evaluated against the LES, and the correlations are nearly 0.97 . The benefit of calculating Stokes drift profiles from directional wave spectra is demonstrated by similarly evaluating three alternatives.
\end{abstract}

\section{Introduction}

The Southern Ocean plays a disproportionately important role in the climate system by taking up more than $40 \%$ of the global ocean's anthropogenic carbon inventory (Khatiwala et al. 2009) and by ventilating a significant fraction of recently warmed deep waters (Purkey and Johnson 2010; Kouketsu et al. 2011). Therefore, it is problematic that ocean general circulation models (OGCMs) have long struggled to represent the Southern Ocean faithfully. In coupled solutions of the Community Earth System Model, for example, the zonal-mean wind stress over the Southern Ocean is much stronger than observed. Nonetheless, ocean mixed layer depth (MLD) is still substantially too shallow in key regions of water mass formation, thus implicating deficiencies in the ocean component

\footnotetext{
Corresponding author: W. G. Large, wily@ucar.edu
}

(Danabasoglu et al. 2012; Weijer et al. 2012). Similar biases remain at eddy-resolving resolutions, suggesting problems with representations of vertical physics (e.g., Fox-Kemper et al. 2011; Belcher et al. 2012). The magnitude of these shallow biases is truly remarkable, exceeding $400 \mathrm{~m}$ in midlatitudes $\left(40^{\circ}-60^{\circ} \mathrm{S}\right)$ during later winter (e.g., Downes et al. 2014; DuVivier et al. 2018), and is the motivation for the present work. It is one part of a larger Southern Ocean project to extend the bias attribution also to surface forcing, the general circulation, the subsurface salinity structure, model resolution and other factors (e.g., DuVivier et al. 2018).

The understanding and hence modeling of Southern Ocean mixing physics has long been plagued by a paucity of observations, but recent observational datasets provide an opportunity for progress. The Southern Ocean Flux Station (SOFS) is part of the Southern Ocean Time Series Observatory of the Australian 
Integrated Marine Observing System (http://imos.org.au/ facilities/deepwatermoorings/sots). It is located in a region of extreme weather events and shallow winter MLD biases, $580 \mathrm{~km}$ southwest of Tasmania near $140^{\circ} \mathrm{E}$, $47^{\circ} \mathrm{S}$. In addition, the ARGO float program ${ }^{1}$ provides high vertical resolution temperature and salinity in the region and throughout the Southern Ocean.

Still missing, however, are direct measurements of turbulent fluxes in the open ocean. Instead, recent research has utilized idealized large-eddy simulation (LES; e.g., McWilliams et al. 1997; Grant and Belcher 2009; Harcourt and D'Asaro 2008; Roekel et al. 2012; $\mathrm{Li}$ and Fox-Kemper 2017). The latter discusses the others in some detail and evaluates schemes for incorporating the effects of Langmuir circulations driven by surface wave induced Stokes drift in OGCMs. Furthermore, Belcher et al. (2012) used some of these LES results, available forcing data and simple energy scaling to argue that "wave-forcing and hence Langmuir turbulence could be important over wide areas of the ocean and in all seasons in the Southern Ocean."

A solid foundation for understanding near surface turbulence is Monin-Obukhov similarity theory (Monin and Obukhov 1954; Wyngaard 2010). However, ocean applications (e.g., Large et al. 1994) rely on empiricism from the atmospheric surface layer, but not directly validated in the ocean. Therefore, similarity theory is reviewed and extended to Stokes forcing of the ocean in section 2. Our LES model, meteorological forcing, and calculations of Stokes drift and of boundary layer depth are described in section 3, where examples of the turbulent solutions provide qualitative validation of the simulations and justification of various configuration choices. In section 4, Southern Ocean LES are used to modify the empirical buoyancy similarity functions for the ocean and to formulate new Stokes similarity functions. A practical parameterization is developed and evaluated in section 5. A similar evaluation of alternatives follows in section 6 , before the discussion and conclusion of section 7 .

\section{Monin-Obukhov similarity theory}

Semiempirical Monin-Obukhov similarity theory is well established in the atmospheric surface layer (Foken 2006). This layer begins at a distance $d_{o}$ from the surface and outside the direct influence of the surface and its

\footnotetext{
${ }^{1}$ ARGO data are collected and made freely available by the International Argo Program and the national programs that contribute to it. (http://www.argo.ucsd.edu, http://argo.jcommops.org). The ARGO Program is part of the Global Ocean Observing System.
}

roughness elements. It extends to a fraction $\varepsilon$ of the planetary boundary layer depth $h$. The theory states that the only parameters (independent variables) governing the structure (dependent variables) of a neutral surface layer are the distance $d$ from the boundary and the kinematic wind stress, $u^{* 2}=\rho^{-1}|\boldsymbol{\tau}|$, where $\tau$ is the surface wind stress, $u^{*}$ is the friction velocity, and $\rho$ is fluid density. Throughout this layer the wind-driven Eulerian flow is generally aligned with the stress, so defining the mean components as $U$, aligned; $V$, orthogonal in the horizontal; and $W$, the upward vertical ( $z$ direction) gives a natural Cartesian coordinate system, where $W$ is zero and $V$ is usually much smaller than $U$. The velocity is then given by $\left\{U+u^{\prime}, V+v^{\prime}, w^{\prime}\right\}$, where $u^{\prime}, v^{\prime}$, and $w^{\prime}$ are fluctuations about the mean $\mathbf{U}=\{U, V, 0\}$.

Dimensional analysis says that dimensionless groups should be constants, and for vertical shear the dependent variable, the empirical von Kármán constant is $\kappa=0.40$. The addition of buoyancy, with mean $\Theta$, fluctuations $\theta^{\prime}$, and surface flux $B_{0}$, to the system adds one more independent variable, $\theta^{*}=B_{0} / u^{*}$, but not a dimension. According to the Buckingham-pi theorem, the nondimensional shear and stratification then become functions of one dimensionless group (Wyngaard 2010), which is traditionally the stability parameter

$$
\zeta=\frac{d}{L}=\frac{\kappa d B_{0}}{u^{* 3}}=\kappa \sigma \frac{\left(-w^{* 3}\right)}{u^{* 3}}
$$

where $\sigma=d / h$ and (1) defines both the Monin-Obukhov depth $L$ and the convective velocity scale $w^{*}$. The surface flux $B_{0}$ is defined to be positive when it stabilizes the surface layer, such that with unstable forcing $\zeta$ and $\theta^{*}$ are negative and $w^{* 3}=\left(-B_{0} h\right)$ is positive. The nondimensional gradients are given by

$$
\begin{gathered}
\Psi_{m}=\frac{\kappa d}{u^{*}} \partial_{z} \mathscr{Q b}=\phi_{m}(\zeta) \\
\Psi_{s}=\frac{\kappa d}{\theta^{*}} \partial_{z} \Theta=\phi_{s}(\zeta),
\end{gathered}
$$

where $\phi_{m}$ and $\phi_{s}$ are empirical functions of $\zeta$. Over land, the vertical shear $\partial_{z} \mathscr{Q b}$ is the magnitude of the vector Eulerian velocity gradient $\left|\partial_{z} \mathbf{U}\right|$.

In the surface layer of the ocean's top boundary layer the presence of Stokes drift $\mathbf{U}_{S}(z)$, gives these nondimensional gradients an additional dependency on empirical Stokes similarity functions, say $\chi_{m}$ and $\chi_{s}$, of a second dimensionless group $\xi$ :

$$
\begin{gathered}
\Psi_{m}=\phi_{m}(\zeta) \chi_{m}(\xi), \\
\Psi_{s}=\phi_{s}(\zeta) \chi_{s}(\xi) .
\end{gathered}
$$


The Stokes parameter $\xi$ contains the important information about the Stokes forcing, such as the turbulent Langmuir number, $\mathrm{La}=\left[u^{*} /\left|\mathbf{U}_{S}(0)\right|\right]^{1 / 2}$ (McWilliams et al. 1997). Also, the Lagrangian velocity becomes $\mathbf{U}_{L}=\mathbf{U}+\mathbf{U}_{S}$, so there are additional options for $\partial_{z} \mathscr{Q b}$ in (2).

The empirical formulations of $\phi_{m}$ and $\phi_{s}$ over land are reviewed by Högström (1988), and for stable forcing

$$
\phi_{m}(\zeta)=\phi_{s}(\zeta)=1+5 \zeta .
$$

Measurements in unstable conditions are typically in the range $-1<\zeta<0$ and give

$$
\begin{gathered}
\phi_{m}(\zeta)=(1-16 \zeta)^{-1 / 4}, \\
\phi_{s}(\zeta)=(1-16 \zeta)^{-1 / 2},
\end{gathered}
$$

though Carl et al. (1973) report better fits at higher altitudes with a $-1 / 3$ exponent.

The search for Stokes similarity functions, $\chi_{m}$ and $\chi_{s}$, is guided by established empiricism and by the basic premise that the structure of the surface layer is determined by the surface forcing, rather than the turbulence within the layer. Indeed, Carl et al. (1973) report that nonneutral Monin-Obukhov scaling, (2) and (3), describe observed profiles well, provided that surface values of scaling parameters $\left(u^{*}\right.$ and $\left.\theta^{*}\right)$ are used. Moving to the ocean, both McWilliams and Sullivan (2000) and Smyth et al. (2002), for example, use the Langmuir number to scale the surface forcing associated with surface waves. Then Roekel et al. (2012) consider misalignment between the wind stress and Stokes drift by up to $135^{\circ}$. They account for the effects in terms of the angle between the axial direction of Langmuir circulation cells and the wind stress, using vertical integrals through the boundary layer, but find that integrating only to $0.2 h$, as suggested by Harcourt and D'Asaro (2008), makes little difference.

\section{a. The turbulent kinetic energy equation}

In general, the directions of both the Stokes drift and the mean current, as well as the angle between them, vary with depth. To deal with high degrees of misalignment, the Stokes drift profile will be regarded as an independent forcing, by considering the kinematic form of the turbulent kinetic energy (TKE) equation. The Craik-Leibovich equation set (Craik and Leibovich 1976), with Stokes drift (McWilliams et al. 1997; Belcher et al. 2012) gives:

$$
\begin{aligned}
\partial_{t} \mathrm{TKE}= & -\left\langle w^{\prime} \mathbf{u}^{\prime}\right\rangle \cdot \partial_{z} \mathbf{U}-\left\langle w^{\prime} \mathbf{u}^{\prime}\right\rangle \cdot \partial_{z} \mathbf{U}_{S}+\left\langle w^{\prime} \theta^{\prime}\right\rangle \\
& + \text { transport }- \text { dissipation, }
\end{aligned}
$$

where \langle\rangle denotes a time and/or horizontal space average. Respectively, the first three terms on the right-hand side represent the production of TKE through the vertical shear of horizontal currents, through the Stokes shear, and by buoyancy. In stable forcing the latter is negative and suppresses TKE. With highly variable meteorological forcing and Stokes drift the dot products of (9) matter and are accounted for by integrating these terms, following Roekel et al. (2012), but just over the surface layer, following the above guidelines. These integrals define the cubes of three velocity scales for the surface layer forcing:

$$
\begin{aligned}
\mu_{U}^{3} & =\int_{-\varepsilon h}^{0}\left[\left(-\left\langle w^{\prime} \mathbf{u}^{\prime}\right\rangle\right) \cdot \partial_{z} \mathbf{U}\right] d z=P_{U} u^{* 3}, \\
\mu_{S}^{3} & =\int_{-\varepsilon h}^{0}\left[\left(-\left\langle w^{\prime} \mathbf{u}^{\prime}\right\rangle\right) \cdot \partial_{z} \mathbf{U}_{S}\right] d z \\
& =P_{S} u^{* 2}\left|\mathbf{U}_{S}(0)\right|=P_{S} \mathrm{La}^{-2} u^{* 3}, \quad \text { and } \\
\mu_{B}^{3} & =\int_{-\varepsilon h}^{0}\left\langle w^{\prime} \theta^{\prime}\right\rangle d z=P_{B} w^{* 3},
\end{aligned}
$$

as well as three associated parameters, $P_{U}, P_{S}$ and $P_{B}$, respectively. Variations in these parameters arise from failures of surface forcing, as given by $u^{*}, \mathrm{La}^{-2}$, and $\left(-B_{0} h\right)$, to account for all the variability in TKE production in the surface layer. Much of this variability is due to misalignment of the stress vector $\left(-\left\langle w^{\prime} \mathbf{u}^{\prime}\right\rangle\right)$, the Stokes shear, and the Eulerian shear. It is captured by the dot products in (10) and (11), which in principle could make $P_{U}$ or $P_{S}$ negative, for example when there are counter inertial currents, or wave components propagating into the wind.

Surfaces fluxes pass through the surface layer to the boundary layer interior and Monin-Obukhov similarity theory provides the viscosity $K_{m}$ and diffusivity $K_{s}$ associated with these transfers, and hence the turbulent Prandtl number, $\operatorname{Pr}=\left(K_{m} / K_{s}\right)$. The theory says that they depend, respectively, on turbulent velocity scales $w_{m}$ and $w_{s}$ :

$$
\begin{aligned}
& K_{m}=w_{m} d ; \quad w_{m}=\frac{\kappa u^{*}}{\phi_{m}(\zeta) \chi_{m}(\xi)}, \\
& K_{s}=w_{s} d ; \quad w_{s}=\frac{\kappa u^{*}}{\phi_{s}(\zeta) \chi_{s}(\xi)}, \quad \text { and } \\
& \operatorname{Pr}=\frac{\phi_{s}}{\phi_{m}} \frac{\chi_{s}}{\chi_{m}} .
\end{aligned}
$$

In the absence of Stokes forcing $\left(\chi_{m}=\chi_{s}=1\right)$ these surface layer velocity scales increase with depth in unstable conditions $\left(\phi_{s}<\phi_{m}<1 ; \operatorname{Pr}<1\right)$ and decrease in stable conditions $\left(\phi_{s}=\phi_{m}>1 ; \operatorname{Pr}=1\right)$. 
Thus, the velocities $w_{m}$ and $w_{s}$ become scales for vertical fluxes in the surface layer and perhaps beyond, and possibly for other turbulent statistics, such as TKE, with the LES providing a means of verification. This scaling is fundamentally different from solving (9) either diagnostically assuming steady state, or prognostically. A classic example of the latter is the Mellor and Yamada (1982) level-2.5 boundary layer, where the velocity scales become proportional to the independent variable, $\mathrm{TKE}^{1 / 2}$. A recent diagnostic example is Chor et al. (2018), where the dissipation in (9) becomes the key independent variable, with $w_{m}^{3}=w_{s}^{3}=$ dissipation times an empirical length scale and hence $\operatorname{Pr}=1$. The different approaches could be closely related when assumptions about equilibrium, the production and dissipation balance, and the relationship of dissipation to TKE are satisfied, as in idealized LES. However, they may differ significantly in nonequilibrium due to variable wind, wave and buoyancy forcing, with misalignment and inertial motions.

In section $4 \mathrm{~b}$, LES of the Southern Ocean boundary layer in April and June are used to extend similarity theory to include Stokes-forced surface layers by formulating $\chi_{m}$ and $\chi_{s}$ to depend on surface forcing and Stokes drift, with $P_{U}, P_{S}$, and $P_{B}$ accounting for misalignment and other sources of variability. This extension does not involve a complete new dimensional analysis with $\mu_{U}^{3}, \mu_{S}^{3}$, and $\mu_{B}^{3}$ as independent variables, because there are no observations to complement and verify the LES, and decades of experience leading to the von Kármán constant as well as to the above $\phi_{m}$ and $\phi_{s}$ functions would be lost. Rather, compatibility with existing No-Stokes empiricism will be maintained through judicious choices of the independent variables.

\section{The large-eddy simulations of the Southern Ocean}

Our LES model, as adapted to simulate ocean boundary layers, is well tested and documented (e.g., Sullivan et al. 2012; Roekel et al. 2012; Kukulka et al. 2013). The model dynamics integrate the wave-averaged, incompressible, and Boussinesq Craik-Leibovich equation set (McWilliams et al. 1997). The subgrid-scale (SGS) fluxes are modeled with the eddy viscosity described in Moeng (1984), Sullivan et al. (1994), and McWilliams et al. (1997). The additional terms arising from phase averaging over the surface waves include: StokesCoriolis, vortex force, and a Bernoulli pressure head in the momentum equations, and horizontal advection by Stokes drift in the scalar equations, as well as additional production of subgrid-scale energy by vertical gradients of Stokes drift (Sullivan et al. 2007). Submesoscale turbulence structures do not develop, because neither mesoscale straining by ocean eddies, nor unstable frontal features are imposed, as in Hamlington et al. (2014), McWilliams (2016), and Sullivan and McWilliams (2018). Wave breaking (e.g., Sullivan et al. 2004, 2007) is not considered. The ocean surface is flat, with a $w^{\prime}=0$ boundary condition, but in contrast to LES over land neither $u^{\prime}=0$, nor $v^{\prime}=0$, are imposed. The distance $d_{o}$ needs to be deeper than the influence of these boundary conditions, as well as other surface influences, so it is determined empirically (section 4).

The ocean LES do not include an explicit salinity, so its thermodynamic variable is buoyancy, $\Theta=g\left(1-\rho / \rho_{o}\right)=$ $g\left[\alpha\left(T-T_{o}\right)-\beta\left(S-S_{o}\right)\right]$, where $g=9.81 \mathrm{~m} \mathrm{~s}^{-2}$ is gravitational acceleration and $T, S$, and $\rho_{o}=1026.6 \mathrm{~kg} \mathrm{~m}^{-3}$ are, respectively, ocean potential temperature, salinity, and density at a typical SOFS autumn surface temperature $T_{o}=12^{\circ} \mathrm{C}$ and salinity $S_{o}=35 \mathrm{psu}$. Thermal expansion is a constant, $\alpha=1.9 \times 10^{-4}{ }^{\circ} \mathrm{C}^{-1}$, and $\beta=$ $7.8 \times 10^{-4}$ is haline contraction. The surface buoyancy forcing $B_{0}$ can include both heat and freshwater fluxes. It is related to an equivalent surface heat flux into the ocean $Q_{0}$ by

$$
B_{0}=\frac{g \alpha}{\left(\rho_{o} C_{p}\right)} Q_{0}
$$

where $C_{p}$ is ocean heat capacity and $\left(\rho_{o} C_{p}\right)=$ $4.1 \mathrm{MJ} \mathrm{m}^{-3} \mathrm{~K}^{-1}$.

Seven large-eddy simulations are documented in Table 1. Four of these mimic two specific time periods at SOFS in April and June of 2010. The two cases of Stokes forcing are denoted as AprS and JunS, and the NoStokes as AprN and JunN. The SOFS air-sea flux mooring (Schultz et al. 2012) provides the meteorological forcing. These simulations are spun up by holding the forcing fixed for $12 \mathrm{~h}$ in order to generate turbulence over the depth of the boundary layer. In the other three cases, D24S, D12S, and D06S, winds are idealized and generate surface waves that are always perfectly aligned, there is a small constant surface buoyancy loss $\left(Q_{0}=-5 \mathrm{~W} \mathrm{~m}^{-2}\right)$, and $h$ is initially $96 \mathrm{~m}$. The wind blows steadily to the east for $12 \mathrm{~h}$, then drops from 20 to $4 \mathrm{~m} \mathrm{~s}^{-1}$ on 24-, 12-, and 6-h time scales, respectively, and afterward remains constant. All seven LES use the SOFS $\left(47^{\circ} \mathrm{S}\right)$ Coriolis parameter, $f=-1.064 \times 10^{-4} \mathrm{~s}^{-1}$, so the inertial period is $16.4 \mathrm{~h}$.

The choice of computational domain $\left\{L_{x}, L_{y}, L_{z}\right\}$, and mesh size $\left\{N_{x}, N_{y}, N_{z}\right\}$ is based on past experience and then confirmed (section 3d) by inspecting the turbulent flow. The horizontal domain needs to be sufficiently 
TABLE 1. Large-eddy simulations of the Southern Ocean in April and June 2010. The respective start dates are 29 Apr and 7 Jun 2010. The start times, $t=0$, correspond to local time in hours (UTC + 0920). The computational domain is $L_{x} \times L_{y}$ in the horizontal and $L_{z}$ in the vertical and covered by a mesh of $N_{x} \times N_{y} \times N_{z}$ grid cells. The vertical grids stretch from a finest resolution of $\Delta Z_{\min }$ at the surface to a maximum $\Delta Z_{\max }$ at the bottom.

\begin{tabular}{lcccccc}
\hline \hline & AprN & JunN & AprS & JunS & D24S & D12S \\
\hline$t=0$ (local time) & 0000 & 0645 & 0000 & 0645 & & \\
Duration (h) & 50 & 38 & 53 & 41 & 100 & 100 \\
$\left\{L_{x}, L_{y}\right\}(\mathrm{m})$ & 1250 & 1250 & 1250 & 1250 & 1000 & 1000 \\
$L_{z}(\mathrm{~m})$ & 300 & 500 & 300 & 500 & 350 & 100 \\
$\left\{N_{x}, N_{y}\right\}$ & 1280 & 1280 & 1280 & 1280 & 1024 & 1000 \\
$N_{z}$ & 512 & 512 & 512 & 512 & 256 & 1024 \\
$\Delta Z_{\min }(\mathrm{m})$ & 0.375 & 0.376 & 0.375 & 0.376 & 0.301 & 256 \\
$\Delta Z_{\max }(\mathrm{m})$ & 1.13 & 2.02 & 1.13 & 2.02 & 1.61 & 0.301 \\
Time step (s) & 0.9 & 1.5 & 0.9 & 1.5 & 0.8 & 1.61 \\
\hline
\end{tabular}

wide to permit multiple coherent largest scale structures to develop independent of the periodic sidewall boundary conditions, while the mesh resolution needs to be sufficiently fine to capture small scales near the surface. Based on atmospheric and oceanic LES (e.g., Moeng and Sullivan 1994; Sullivan and Patton 2011; Sullivan et al. 2012) the horizontal domain should be about 5 times the boundary layer depth to minimize the influence of periodic sidewalls. The vertical extent of the domain $L_{z}$ is about twice $h$ to permit a smooth transition between the turbulent and stably stratified layers. The vertical grid is stretched algebraically with the ratio between neighboring cell thicknesses always less than 1.0035 . Its resolution is finest, $\Delta Z_{\min }$, at the top to resolve the rapid vertical decay of the Stokes drift profile, with 5 points over the $e$-folding depth. Experience shows that the subgrid-scale model in LES tolerates a small amount of anisotropy, $L_{x} / N_{x}<3 \Delta Z_{\text {min }}$ (Sullivan et al. 2003). All these considerations lead to the domain choices in Table 1, which then give the maximum cell thickness $\Delta Z_{\max }$ at the bottom and sets the time step.

\section{a. Varying Southern Ocean surface conditions}

The highly variable LES forcing is highlighted by overlapping the time series in Fig. 1. In April (blue traces), high winds $\left(>20 \mathrm{~m} \mathrm{~s}^{-1}\right)$ persisted for nearly a day prior to $t=0$ (Table 1), so there are large-amplitude waves with significant wave height $H_{S} \sim 9 \mathrm{~m}$. Afterward there are six distinct forcing regimes that are characterized in Table 2. During the first regime, denoted A1-11 for April hours 1-11, the wind is strong $\left(u^{*}>\right.$ $0.02 \mathrm{~m} \mathrm{~s}^{-1}$; Fig. 1a), steady (Fig. 1d), and aligned with the surface Stokes drift (Fig. 1c), and there is substantial buoyancy loss (Fig. 1e). The wind weakens over next $4 \mathrm{~h}$ (A12-15; Fig. 1a), and there is significant diurnal variation in $Q_{0}$. The wind speed drops to $4 \mathrm{~m} \mathrm{~s}^{-1}$ in the third regime, A16-22, while the rise of $\mathrm{La}^{-2}$ to 15 (Fig. 1d), indicates that the waves $\left(H_{S} \sim 5 \mathrm{~m}\right)$ remain stronger than wind-wave equilibrium. Over the next $9 \mathrm{~h}, \mathrm{~A} 23-31$, the winds are light, highly variable in direction, and hence greatly misaligned with the waves. The next regime A32-43 is characterized by a night-day-night transition $\left(-10<Q_{0}<250 \mathrm{~W} \mathrm{~m}^{-2}\right)$, and includes all the stable forcing. The winds of the final April regime, A44-50, rise from about 6 to $15 \mathrm{~m} \mathrm{~s}^{-1}$ and blow increasingly toward the south, while the buoyancy forcing remains near neutral $\left(\left|Q_{0}\right|<30 \mathrm{~W} \mathrm{~m}^{-2}\right)$.

In the June case (Fig. 1; magenta), the simulations begin just after a wind and wave lull, so the moderate buoyancy loss is a major surface forcing initially and over the first June forcing regime, J1-4. Three subsequent regimes are also characterized in Table 2 . As the wind increases to more than $15 \mathrm{~m} \mathrm{~s}^{-1}$ over the next $23 \mathrm{~h}$ (J5-27), a local wave field develops and stays near equilibrium $\left(\mathrm{La}^{-2} \approx 11\right.$ ), with $H_{s} \sim 5 \mathrm{~m}$, while the buoyancy forcing is modulated by daytime solar heating. Over the next regime, J34-41, $Q_{0}$ becomes very close to zero, because of the solar heating. During the last June regime, J34-41, the wind weakens with highly variable direction and misalignment.

The wind speed decay (e.g., Fig. 1a; red) gives three regimes in each of the idealized cases; D1-26 until the peak in $\mathrm{La}^{-2}$, D41-100 when the weak winds permit significant inertial motions to persist, and a transition regime D27-40.

\section{b. Stokes drift profiles}

Time-varying wave effects are generated by the wave prediction model, WAVEWATCH III (Tolman 2002). The model physics (Romero and Melville 2010b) are based on the work by Alves et al. (2003) forcing the high frequencies to match the empirical degree of spectral saturation (Romero and Melville 2010a) and on "exact" computations of the nonlinear wave-wave resonant 

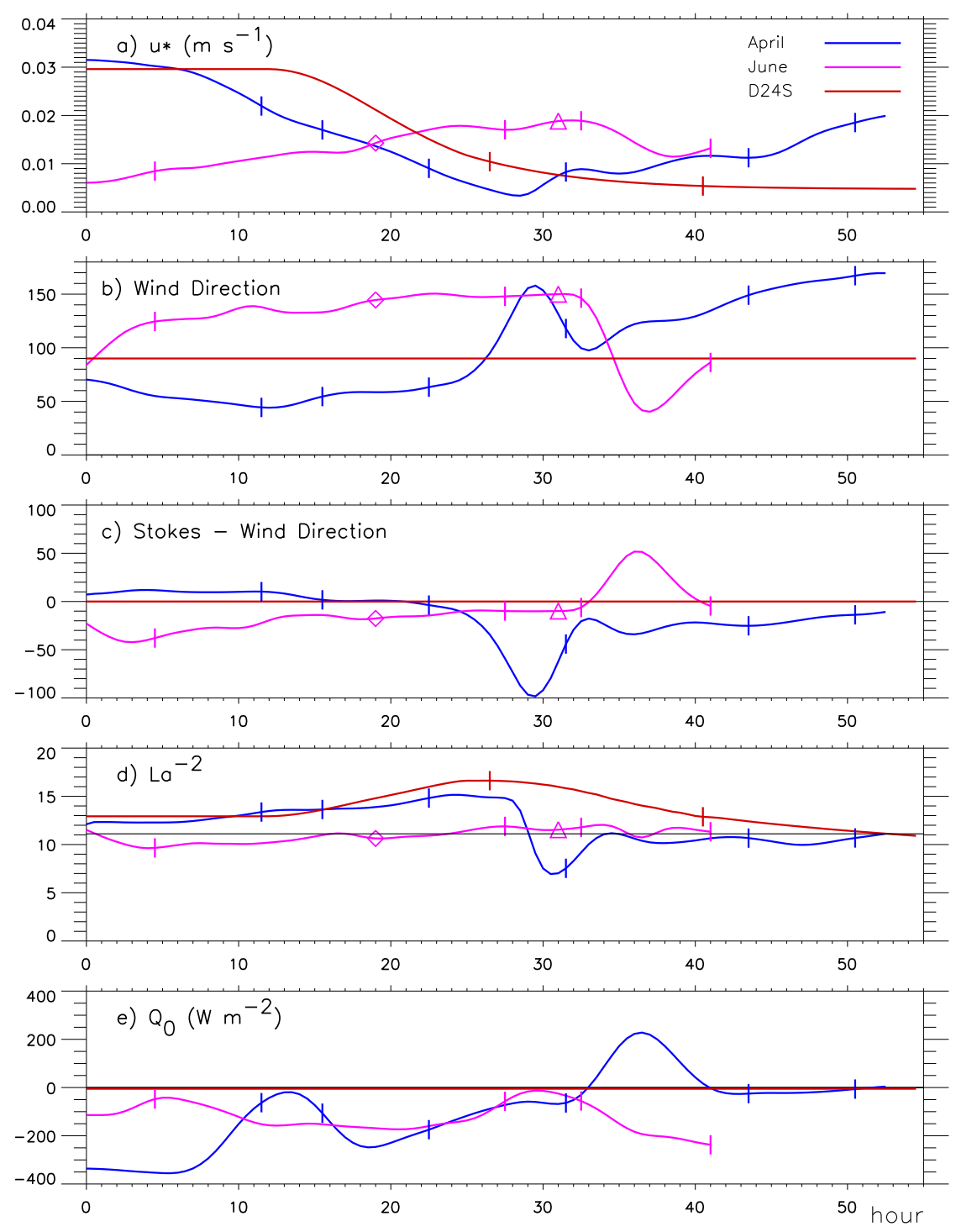

FIG. 1. LES surface forcing from 2330 local time 28 Apr 2010 (blue), from 0615 local time 7 Jun 2010 (magenta), and as idealized for D24S (red): (a) friction velocity $u^{*}$; (b) the direction to which the wind blows in degrees from north; (c) the difference in the direction of the surface Stokes drift and the wind; (d) $\mathrm{La}^{-2}$, the ratio of the speed of the surface Stokes drift to $u_{*}$, with a black line at the wind-wave equilibrium value of $\mathrm{La}^{-2}=11$ (McWilliams et al. 1997); and (e) equivalent surface heat flux $Q_{0}$. The vertical bars demarcate the forcing regimes of Table 2. The surface wave fields of Fig. 2 are from the times of the magenta diamond and triangle.

interactions (van Vledder 2006). The model is driven by winds of Fig. 1a, using the formulation by Snyder et al. (1981). This local wind forcing is combined with spectral wave data from ECMWF ERA-Interim global reanalysis (Berrisford et al. 2011) to better capture the low wavenumber/frequency components of the wave spectrum. The ECMWF wave spectra with a horizontal resolution of $0.75^{\circ}$ are used to provide boundary and initial conditions for WAVEWATCH III in a model domain 9 grid points in longitude by 12 in latitude centered at the SOFS mooring, with a resolution of $0.25^{\circ}$. The spectral grid is composed of 55 frequencies $\varpi$ between 0.0310 and $2.3 \mathrm{~Hz}$ with a logarithmic resolution $\Delta \varpi / \varpi=0.083$ and 72 azimuthal grid points $\varphi$. The wave model integration time step is $150 \mathrm{~s}$. The combined directional wave height spectra $F(\varpi, \varphi, t)$ are used to generate time varying vertical profiles of the Stokes drift according to 
TABLE 2. Characteristics of the April, June, and idealized decay forcing regimes from $t_{1}$ to $t_{2}$ (h) from time $t=0$ (Table 1 ), with ranges when there is significant variability. The right-most column gives the empirical shallow limit of the surface layer $d_{o}$ (section 4 ), with both No-Stokes (N) and Stokes forcing (S).

\begin{tabular}{|c|c|c|c|c|c|c|c|c|c|}
\hline \multirow[b]{2}{*}{ Forcing regime } & \multirow[b]{2}{*}{$t_{1}$} & \multirow[b]{2}{*}{$t_{2}$} & \multirow[b]{2}{*}{ Wind speed $\left(\mathrm{m} \mathrm{s}^{-1}\right)$} & \multirow{2}{*}{$\begin{array}{l}\text { Wind direction } \\
\left({ }^{\circ} \text { from } \mathrm{N}\right)\end{array}$} & \multirow[b]{2}{*}{$Q_{0}\left(\mathrm{~W} \mathrm{~m}^{-2}\right)$} & \multirow[b]{2}{*}{$\mathrm{La}^{-2}$} & \multirow{2}{*}{$\begin{array}{l}\text { Stokes direction } \\
\left({ }^{\circ} \text { from wind }\right)\end{array}$} & \multicolumn{2}{|c|}{$d_{o}(\mathrm{~m})$} \\
\hline & & & & & & & & $\mathrm{N}$ & $\mathrm{S}$ \\
\hline A1-11 & 1 & 11 & $20 \rightarrow 16$ & 60 & $-350 \rightarrow-100$ & 13 & 10 & 3.0 & 3.8 \\
\hline A12-15 & 12 & 15 & $16 \rightarrow 10$ & 50 & -50 & 14 & 5 & 2.6 & 3.0 \\
\hline A16-22 & 16 & 22 & $10 \rightarrow 6$ & 60 & $-100 \rightarrow-250$ & 14 & 0 & \multicolumn{2}{|c|}{$2.3^{.0}$} \\
\hline A23-31 & 23 & 31 & $6 \rightarrow 3$ & $75 \rightarrow 160$ & $-150 \rightarrow-50$ & $15 \rightarrow 7$ & $-20 \rightarrow-100$ & \multicolumn{2}{|c|}{1.5} \\
\hline A $32-43$ & 32 & 43 & $6 \rightarrow 8$ & $100 \rightarrow 140$ & $-50 \rightarrow 240$ & $7 \rightarrow 11$ & -25 & \multicolumn{2}{|c|}{0.8} \\
\hline A44-50 & 44 & 50 & $8 \rightarrow 12$ & $140 \rightarrow 170$ & -30 & 10 & -20 & \multicolumn{2}{|c|}{2.3} \\
\hline $\mathrm{J} 1-4$ & 1 & 4 & 5 & $85 \rightarrow 125$ & -100 & 10 & $-40 \rightarrow-15$ & 3.0 & 1.5 \\
\hline $\mathrm{J} 5-27$ & 5 & 27 & $6 \rightarrow 12$ & 135 & $-50 \rightarrow-150$ & 11 & -15 & 3.0 & 2.3 \\
\hline $\mathrm{J} 28-32$ & 28 & 32 & 13 & 150 & -10 & 12 & -10 & 3.0 & 1.5 \\
\hline $\mathrm{J} 33-41$ & 33 & 41 & $13 \rightarrow 7$ & $150 \rightarrow 40$ & $-15 \rightarrow-250$ & 11 & $10 \rightarrow 50$ & 3.0 & 1.5 \\
\hline D1-26 & 1 & 26 & $20 \rightarrow 7$ & 90 & -5 & $13 \rightarrow 18$ & 0 & \multicolumn{2}{|c|}{1.9} \\
\hline D27-40 & 27 & 40 & $7 \rightarrow 4$ & 90 & -5 & $18 \rightarrow 13$ & 0 & \multicolumn{2}{|c|}{3.0} \\
\hline D41-100 & 41 & 100 & 4 & 90 & -5 & $13 \rightarrow 11$ & 0 & \multicolumn{2}{|c|}{3.8} \\
\hline
\end{tabular}

$$
\mathbf{U}_{S}(z, t)=2 \int_{0}^{\infty} \int_{-\pi}^{\pi} \mathbf{k} \varpi F(\varpi, \varphi, t) e^{2|\mathbf{k}| z} d \varphi d \varpi
$$

where $\mathbf{k}$ denotes the horizontal wavenumber vector.

The Stokes drift forcing fields for the idealized cases were similarly generated with WAVEWATCH III, but assuming an unlimited fetch (i.e., single grid point) forcing the wave spectrum with the idealized time varying wind.

Two examples of directional wave spectra and Stokes drift profiles are shown in Fig. 2 to illustrate how these calculations account not only for transient wind forcing, but also for remotely generated swell. In the first, at hour 19 of the June cases, the broad spectral peak is due to low-frequency swell propagating to east-northeast (Fig. 2a) that gives the Stokes drift below $20 \mathrm{~m}$ (Fig. 2c). Above, the local wind waves developing in response to increasing wind to the southeast, cause the Stokes drift to veer toward the wind direction, though at the surface there is still a misalignment of about $20^{\circ}$. In the second example, from $12 \mathrm{~h}$ later, the still increasing winds reduce this misalignment, deepen the veering (Fig. 2d), and broaden the spectral peak in both direction and frequency (Fig. 2b). At both times the veering of the Stokes shear is well resolved by 25 grid points in the upper $10 \mathrm{~m}$ and captured in $\mu_{S}^{3}$ by the dot product in (11).

\section{c. Boundary layer depths}

Beginning about $11 \mathrm{~h}$ prior to the start of the April LES, there are seven ARGO profiles to 300-m depth at intervals of about $8 \mathrm{~h}$ (roughly half an inertial period), and distances from 7 to $30 \mathrm{~km}$ from SOFS. These profiles provide the LES initial conditions and some validation (section $2 \mathrm{~d}$ ). For both purposes consecutive profiles are averaged to reduce adiabatic signals due to inertial motions.

A unique feature of our LES is the deep depth of maximum stratification $h_{i}$ at the inflection point of the buoyancy profile, which is the analog of the atmospheric inversion. It is mostly set by the initial conditions as is evident in the profile in Fig. 3 a from AprS. Over all four April and June cases, $h_{i}$ deepens only by $4-5 \mathrm{~m}$. Although this deepening is not rapid, the rates are more than twice the roughly $40 \mathrm{~m}$ of ARGO observed deepening over the 40 days between the April and June time periods. The blue trace of Fig. $3 b$ shows the profile of LES buoyancy change over an inertial period from hour 0.5 to 17 of AprS. Most of the buoyancy loss above $168 \mathrm{~m}$ is through the surface, but about one-third is due to entrainment of dense water from deeper, as seen in the buoyancy gain between 170 and $190 \mathrm{~m}$. This entrainment deepens the boundary layer by a few meters from the value of $h>h_{i}$ shown for the A3 profile of Fig. 3c.

An important quantity derived from 1-h LES statistics is a boundary layer depth $h$ to which surface-forced turbulence penetrates. It is calculated from the profiles of buoyancy flux and an unstable example from hour 3 of AprS, is shown by the blue profile (A3) of Fig. 3c. There are three steps: first, find the entrainment depth $h_{e}$ (blue asterisk at $165 \mathrm{~m}$ ), where $\left\langle w^{\prime} \theta^{\prime}\right\rangle_{e}$ is the column minimum above $h_{i}$; second, find the first depth $h_{\max }$ below $h_{e}$ where $\left\langle w^{\prime} \theta^{\prime}\right\rangle_{\max }$ is a relative maximum; and finally, take $h$ as the first depth $(182 \mathrm{~m})$ below $h_{e}$ and above $h_{\max }$, where the buoyancy flux equals the weighted average $(1-0.95)\left\langle w^{\prime} \theta^{\prime}\right\rangle_{e}+0.95\left\langle w^{\prime} \theta^{\prime}\right\rangle_{\max }$. This algorithm has proven to be robust in the sense that a 

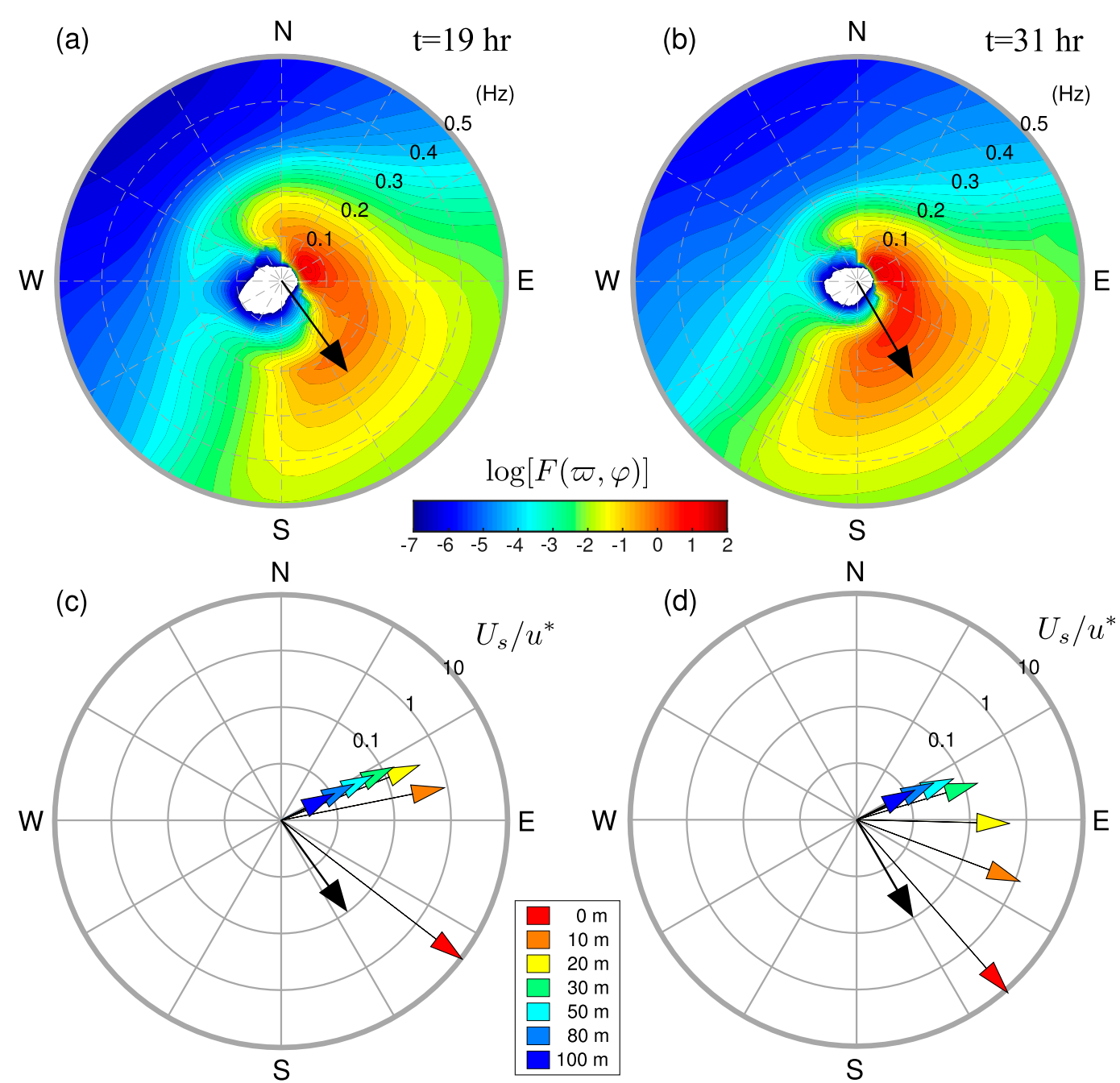

FIG. 2. Combined local plus low-frequency wave states at SOFS on 8 Jun 2010: (a) logarithm of the spectral power $F\left(\mathrm{~m}^{2} \mathrm{~s} \mathrm{rad}^{-2}\right)$, at hour 19 of JunS; (b) as in (a), but for hour 31; (c) Stokes drift vectors $\mathbf{U}_{S}$ at hour 19 corresponding to (a), with magnitudes normalized by $u^{*}$ on a $\log$ scale at depths down to $100 \mathrm{~m}$, where the magnitude of the surface (red) Stokes drift is $\mathrm{La}^{-2}$; and (d) Stokes drift vectors at hour 31, corresponding to (b). The black arrows are in the direction of the observed SOFS wind.

value for $h$ is always found, there is never significant momentum or buoyancy flux below, and it is insensitive to the somewhat arbitrary weight, 0.95 . Also, it is applied throughout the transition regime A32-43, as shown by the red profiles of Fig. 3c. When the buoyancy flux first increases with depth (e.g., A34 and A38), $h_{e}$ is taken to be at the surface, and $h$ shoals from 31 to about $21 \mathrm{~m}$. Later in the afternoon, at A40 for example, the reduced solar heating makes the buoyancy flux near the surface less negative than found deeper, so there is a calculated entrainment depth at about $12 \mathrm{~m}$ and boundary layer depth again at about $31 \mathrm{~m}$. Usually, the algorithm could be simplified by taking $\left\langle w^{\prime} \theta^{\prime}\right\rangle_{\max }$ to be zero. The exceptions include the A34 and the earlier unstable profiles of the transition regime, A32-43, where $\left\langle w^{\prime} \theta^{\prime}\right\rangle_{\max }$ is positive. Otherwise, the shallowing of $h$ is very abrupt.

\section{d. The LES solutions at SOFS}

The wind, wave, and buoyancy forcing are all stronger and more highly variable than in most LES studies. This forcing combined with the deep boundary layer presents an unusual challenge for the model to capture all the turbulence structures as well as their interactions. Although the LES need only mimic, but not necessarily reproduce nature, there are three basic issues to address.

First, do the LES reproduce at least the overall character of the observed time evolution of the upper ocean at SOFS in the autumn of 2010? The ARGO 


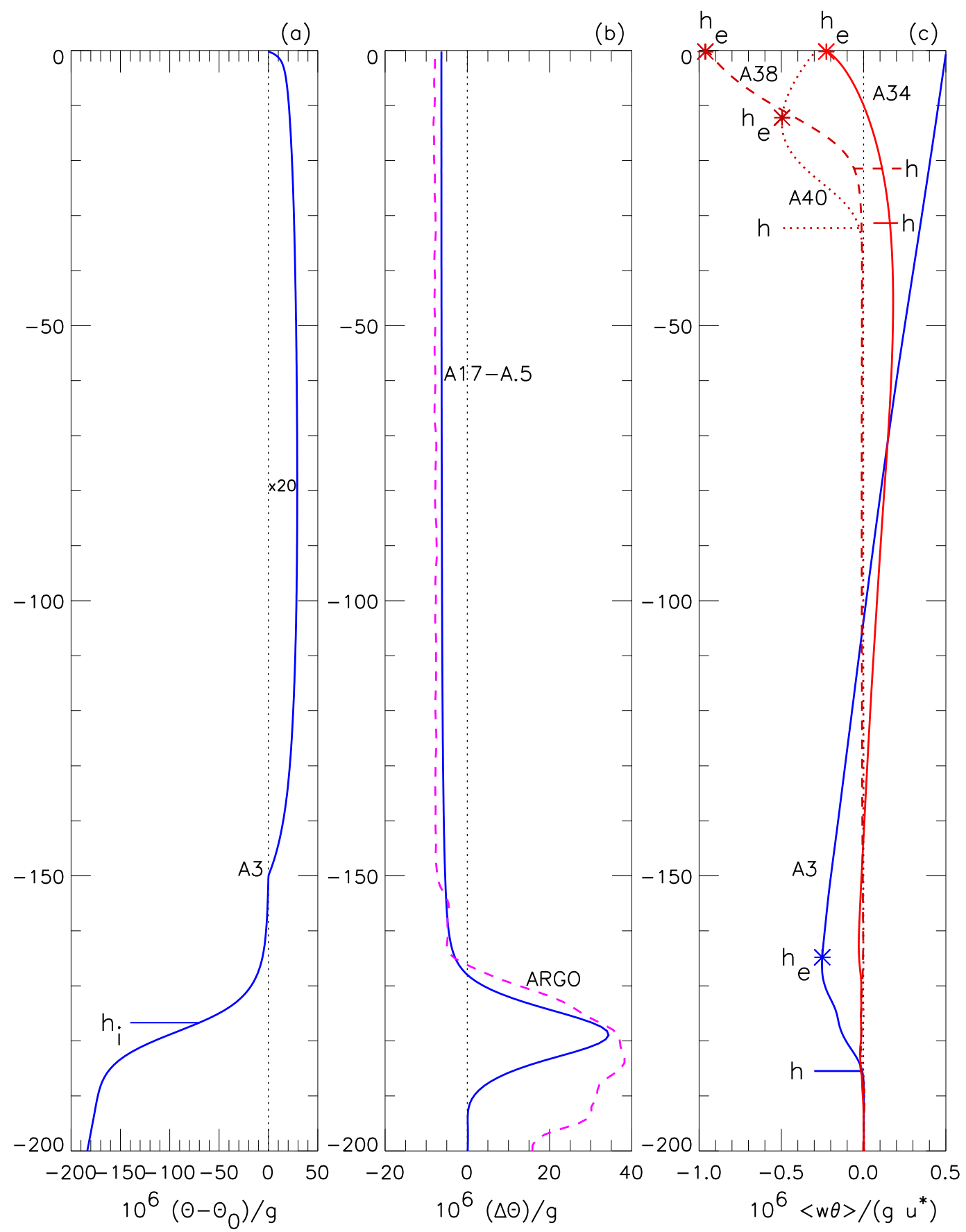

FIG. 3. Vertical profiles from AprS of (a) buoyancy difference from the surface at hour 3, normalized by $g$ with positive values multiplied by 20 for clarity, where $h_{i}$ is the inflection (inversion) depth of maximum stratification; (b) buoyancy change over an inertial period from hour 0.5 to hour 17 , normalized by $g$ from the LES (blue) compared to ARGO (dashed magenta), as described in the text; and (c) buoyancy flux normalized by $g u^{*}$ from the unstable forcing of hour 3 (blue) and from the stable forcing (red) of hours 34 (solid), 38 (dashed), and 40 (dotted), showing the diagnosed entrainment depths $h_{e}$ (asterisk) and boundary layer depths $h$. 
profiles suggest that they do quite well, though even the relatively frequent April sampling is very far from ideal. The ARGO float moves about $36 \mathrm{~km}$ in $46 \mathrm{~h}$, and the ocean responds to real not parameterized forcing and to 3D advection not in the LES. Furthermore, the ARGO profiles indicate that there is a very large adiabatic signal from the semidiurnal internal tide that is missing in the LES. Although undersampled, the phase is such that the signal is minimized by differencing the consecutive average profile ending at hour 3 , from the consecutive average ending at hour 19, with the result shown in Fig. 3b (dashed magenta). The nonzero difference at $200 \mathrm{~m}$ continues past $275 \mathrm{~m}$ and is consistent with lateral advection, or float travel through lateral gradients, or a tidal remnant. Compared to the LES (blue trace), the greater ARGO buoyancy loss above $150 \mathrm{~m}$ could include any combination of the above possibilities, including the different time intervals, or too little LES entrainment, with no way to discriminate. Nonetheless, both differences show buoyancy gain (entrainment) starting at about $168-\mathrm{m}$ depth and peaking near $180 \mathrm{~m}$, where the ARGO change is only about $10 \%$ greater than that from AprS.

Second, are the scales of motion generated by this forcing and their interactions resolved by the computational domains and grids (Table 1)? Flow visualization of the turbulent solutions provides a means of observing the dominant features. Figure 4 is an example of vertical velocity in the lower surface layer at $18 \mathrm{~m}$. At this time (hour 19 of JunS) the winds and buoyancy flux are moderate, but the waves are complicated (Fig. 2a). There are multiple spatially elongated coherent downwelling (blue) structures aligned roughly in the direction of the wind (black vector at the top left). Such features are familiar from Langmuir turbulence associated with surface wave and wind coupling, even though the Stokes drift at this depth is nearly orthogonal (Fig. 2b). The structures are typically about $100 \mathrm{~m}$ wide, up to $800 \mathrm{~m}$ long, and separated by more than $300 \mathrm{~m}$. In contrast Roekel et al. (2012) show much smaller and closer upwelling features ( $10 \mathrm{~m}$ wide, $200 \mathrm{~m}$ long, and $20 \mathrm{~m}$ apart), and roughly symmetric downwelling from their idealized LES. Larger scales in Fig. 4 are expected because of the deeper boundary layer. However, the different character of the upwelling (red), in particular its greater area, and hence the asymmetry of the $w^{\prime}$ distribution, is characteristic of convective cells. These cells are coherent over several hundred meters. Thus, the large domains of Table 1 appear to be necessary and sufficient. They also improve the convergence of the hourly LES statistics.

Third, is the range of LES forcing representative of the SOFS site in autumn? The combined wind and

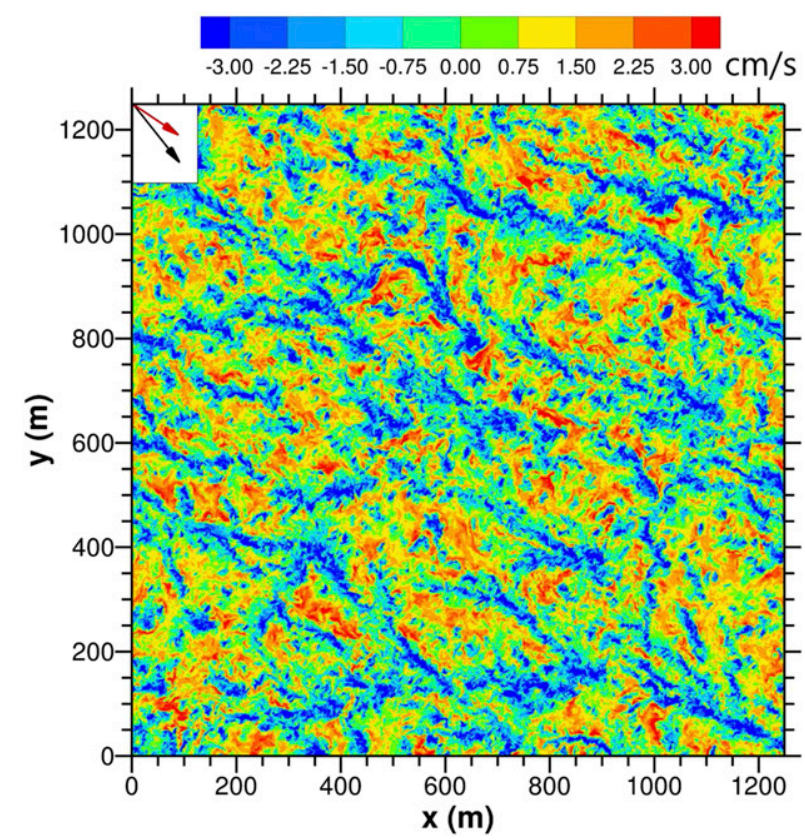

FIG. 4. Instantaneous horizontal section at 18-m depth of vertical velocity, $w^{\prime}$, at hour 19 of JunS. Arrows in the direction of the wind (black) and the surface Stokes drift (red) from Fig. 2c are shown at the top left.

buoyancy forcing during regime A1-11 is the strongest in the SOFS record for April, while A23-43 is among the weakest. The frequency of occurrence distribution created by Belcher et al. (2012) for unstable conditions in Southern Ocean winter is a function of $\mathrm{La}$ and $h / L_{L}=w^{* 3} /\left(\mathrm{La}^{-2} u^{* 3}\right)$. It is distributed narrowly in the range $5<\mathrm{La}^{-2}<20$ and broadly across six orders of magnitude of $h / L_{L}<1000$, with a peak near $\left(\mathrm{La}^{-2}=\right.$ $11, h / L_{L}=0.2$ ). More specifically at SOFS, $\mathrm{La}^{-2}$ exceeds a value of 8 more than $80 \%$ of the time from June through August. Thus, the 7-16 range of $\mathrm{La}^{-2}$ in Fig. 1d shows that the relative strength of Stokes to wind forcing in Fig. 1 is representative of most of the Southern Ocean, particularly at SOFS in autumn. The No-Stokes cases are in the very different regime of $\mathrm{La}^{-2}=0$. The forcing of Fig. 1 also spans the range of Southern Ocean $h / L_{L}$. Unstable values nearly reach 1000 for a few hours of A23-31, with $h / L_{L}<1$ throughout D1-26 of D24S, for example. Negative values come from the stable forcing of regime A32-43.

\section{Similarity functions in the LES surface layer}

The nondimensional gradients $\Psi_{m}$ from (2) and $\Psi_{s}$ from (3) are computed from the meteorological forcing and the LES property gradients. If these gradients are sufficiently in quasi-equilibrium with the forcing for similarity theory to hold in the surface layer, then 
between $d_{o}$ and $\varepsilon h$ they should behave according to (4) and (5), respectively.

With only $8 \mathrm{~h}$ of stable forcing, (6) is assumed to hold for the ocean LES. However, the two No-Stokes cases are used in section $4 \mathrm{a}$ to adapt the unstable buoyancy similarity functions, $\phi_{m}$ from (7) and $\phi_{s}$ from (8). For this purpose the forcing regime restrictions are the most stringent, because considerable insight is available from the atmosphere and because a wind-forced ocean is never waveless. In section $4 b$, the Stokes LES are used to formulate Stokes similarity functions, $\chi_{m}$ and $\chi_{s}$. The Stokes forcing allows the restrictions to be relaxed and stable conditions included. In practice, parameterizations are applied in all conditions, so restrictions are further relaxed for the developments of section 5 , and only a few hours are excluded from the evaluations of section $5 \mathrm{a}$ and section 6 .

\section{a. The $\phi_{m}$ and $\phi_{s}$ similarity functions in the No-Stokes LES}

Only two periods with $Q_{0}<-40 \mathrm{~W} \mathrm{~m}^{-2}$ and relatively steady winds are analyzed; namely, the first $22 \mathrm{~h}$ of AprN (regimes A1-11, A12-15, A16-22) and the first $27 \mathrm{~h}$ of JunS (regimes J1-4 and J5-27). To span the surface layer, $\Psi_{m}$ and $\Psi_{s}$ are computed at three fixed depths $(3.4,4.2$, and $5.0 \mathrm{~m})$, as well as near $\sigma=d / h=0.04,0.06$, $0.08,0.10,0.12$, and 0.14 . Thus, the region nearer the surface where SGS fluxes may become important is avoided.

The functions $\phi_{m}(d / L)$ and $\phi_{s}(d / L)$ are computed from (7) and (8), respectively, at all nine depths. The resulting nondimensional products $\left(\Psi_{m} \phi_{m}^{-1}\right)$ and $\left(\Psi_{s} \phi_{s}^{-1}\right)$ are shown as functions of $\sigma$ in Figs. 5a and 5b, respectively. Although (2) and (3) say they should both equal unity, the former is clearly biased low, but the latter is biased high. Such opposing biases cannot be reconciled through the von Kármán constant. However, Figs. $5 \mathrm{c}$ and $5 \mathrm{~d}$ show that they can be reconciled with $\kappa=0.4$ by adopting the unstable functions

$$
\begin{gathered}
\phi_{m}(\zeta)=(1-14 \zeta)^{-1 / 3}, \\
\phi_{s}(\zeta)=(1-25 \zeta)^{-1 / 3},
\end{gathered}
$$

where the $-1 / 3$ exponents are consistent with Carl et al. (1973) and Frech and Mahrt (1995). The coefficients 14 and 25 are determined from $\sigma$ in the range between 0.02 and 0.10 , where there are 467 data points and the averages of $\phi_{m}$ and $\phi_{s}$ are both 1.00. The respective standard deviations are about 0.12 (Fig. 5c) and 0.20 (Fig. 5d). In the convective limit of no wind and no waves, the $-1 / 3$ exponents give turbulent velocity scales proportional to $w^{*}$, and the coefficients give $\operatorname{Pr}=0.82$.
The points, especially in Fig. 5d, become more scattered and smaller on average for $\sigma>0.1$, suggesting $\varepsilon=0.1$ as the farthest extent of the surface layer. At smaller values of $\sigma$, high biases begin to develop and this tendency is used to determine the nearer extent, $d_{o}$, empirically. The dependency of $d_{o}$ on the forcing regime is shown Table 2, for both No-Stokes and Stokes cases. It is always found to be less than $4 \mathrm{~m}$. It is most variable for AprS when it shallows from $3.8 \mathrm{~m}$ to less than a meter in the transition regime (A32-43), then deepens to $2.3 \mathrm{~m}$ in the near-neutral regime (A44-50). There is less variability without surface wave complications. For example, $d_{o}$ is $3.0 \mathrm{~m}$ throughout JunN. It is the same for both shear and stratification, except during the initially strong winds of D1-26, when the turbulent flow may not still be equilibrated with the initial deep inversion and the respective values are 1.9 and $0.3 \mathrm{~m}$.

\section{b. The $\chi_{m}$ and $\chi_{s}$ functions in the Stokes LES}

Compatibility with No-Stokes empiricism constrains the choices of independent variables for the dimensional analysis of the wind-, wave-, and buoyancy-forced ocean boundary layer. Judicious choices include distance $d$ and buoyancy $\theta^{*}$ as in section 2, plus an additional Stokes velocity, say $V_{\text {Stokes }}$. The velocity variable, say $\mu$, associated with the wind forcing must become $u^{*}$ for $\mathrm{La}^{-2}=0$, so a general form is $\mu=u^{*}\left(1+R \mathrm{La}^{-2}\right)^{p}$, where both the factor $R$ and the exponent, as well as $V_{\text {Stokes}}$, are to be determined empirically from the LES solutions.

The additional factor, $\left(1+R \mathrm{La}^{-2}\right)^{p}$ can be incorporated into the Stokes similarity functions, $\chi_{m}$ and $\chi_{s}$, such that (4) and (5) still define $\Psi_{m}$ and $\Psi_{s}$, respectively. However, both these nondimensional gradients now become functions of two dimensionless groups $\left(d \theta^{*} \mu^{-2}\right)$ and $\left(V_{\text {Stokes }} \mu^{-1}\right)$. The first of these groups suggests modifying the stability parameter $\zeta$ by a factor $(1+$ $\left.R \mathrm{La}^{-2}\right)^{p}$, where $R$ and $p$ are chosen to minimize the depth dependencies of $\Psi_{m}$ and $\Psi_{s}$ that are not captured by the $\phi_{m}$ and $\phi_{s}$ functions of $\zeta$ from (1). Particularly useful for this purpose is regime D1-26 of D24S, because the waves and wind are always aligned, the buoyancy is only weakly unstable, while the wind is initially very strong before falling smoothly to about $6 \mathrm{~m} \mathrm{~s}^{-1}$ (Fig. 1). Also analyzed here are all the regimes of Fig. 5 plus A26-33 where Stokes forcing appears to overcome the highly variable wind direction.

In (1), $\zeta$ is proportional to the ratio $\left(-w^{* 3} / u^{* 3}\right)$, so Stokes effects are included by modifying the denominator. The addition of $u^{* 2}\left|\mathbf{U}_{S}(0)\right|$, leads to $R=1$, adding $\mu_{S}^{3}$ gives $R=P_{S}$, while replacing $u^{* 3}$ with $\left(\mu_{U}^{3}+\mu_{S}^{3}\right)$ leads to $R=\left(P_{S} / P_{U}\right)$. A denominator of $\left(\mu_{U}^{3}+\mu_{S}^{3}+\mu_{B}^{3}\right)$ 

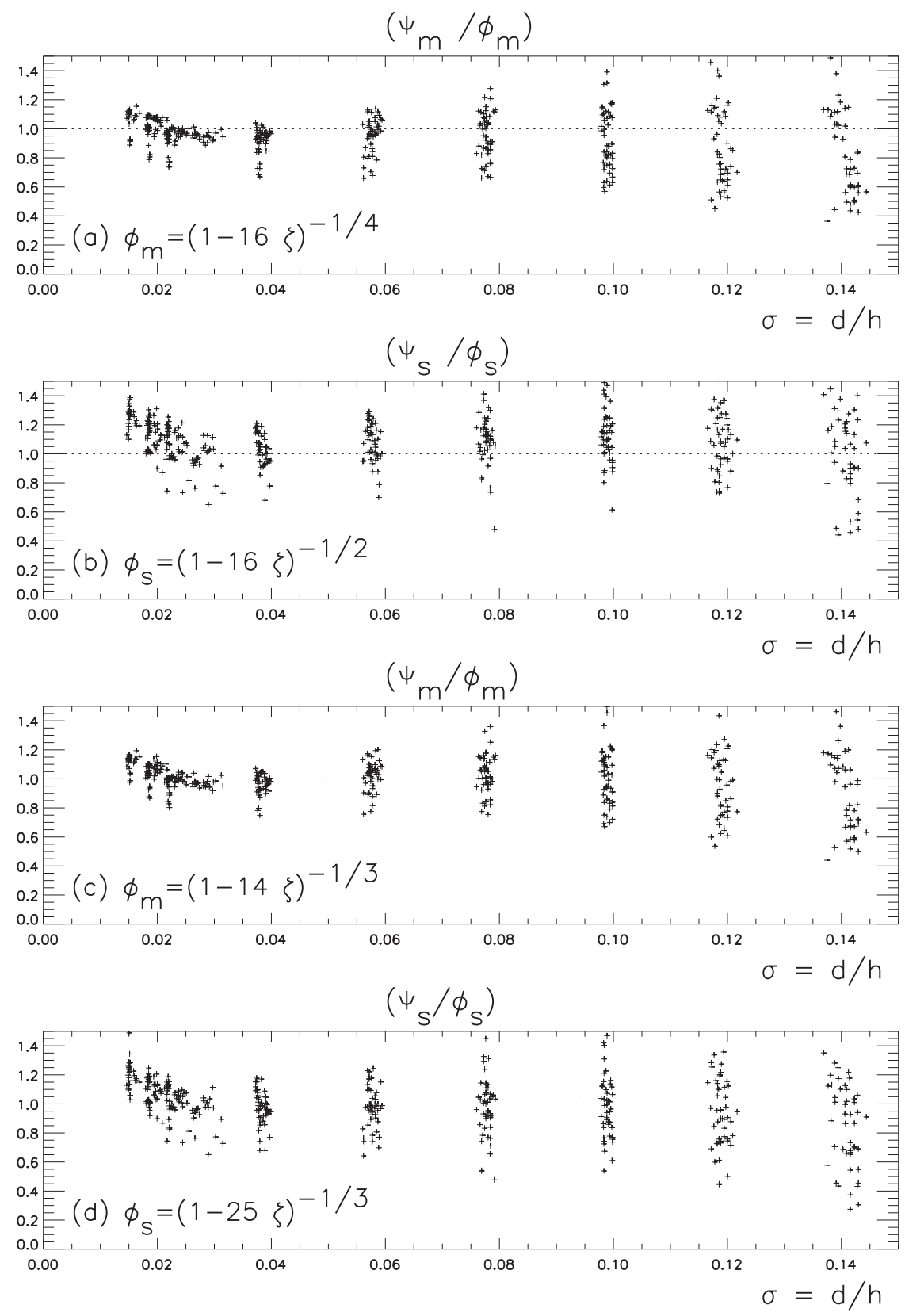

FIG. 5. Results from 49 one-hour averages of unstable forcing from regimes A1-11, A12-15, and A16-22 of AprN and regimes J1-4 and J5-27 of JunN (Table 2) at three fixed depths $(3.4,4.2$, and $5.0 \mathrm{~m})$ and near six fractions $\sigma=0.04,0.06,0.08,0.10,0.12$, and 0.14 of the diagnosed boundary layer depth: (a) $\Psi_{m} \phi_{m}^{-1}$, with $\phi_{m}$ from (7); (b) $\Psi_{s} \phi_{s}^{-1}$, with $\phi_{s}$ from (8); (c) $\Psi_{m} \phi_{m}^{-1}$, with $\phi_{m}=(1-14 \zeta)^{-1 / 3}$; and (d) $\Psi_{s} \phi_{s}^{-1}$, with $\phi_{s}=(1-25 \zeta)^{-1 / 3}$, for $\zeta=d / L(1)$.

is inconsistent with (1) for No-Stokes. To explore consistent options for $p$ between \pm 1 , the integrals of (10), (11), and (12) are solved numerically, using the discrete LES velocities and fluxes to integrate from the first model level below $\varepsilon h$ up to a depth of $\left(\Delta Z_{\min } / 2\right)$. Over the range $-1<p<1$, the least depth dependency is found with $R=\left(P_{S} / P_{U}\right)$ and $p=1 / 2$.
Using these choices, $\chi_{m}=\left(\Psi_{m} \phi_{m}^{-1}\right)$ and $\chi_{s}=\left(\Psi_{s} \phi_{s}^{-1}\right)$ are computed at all depths down to $\sigma=0.25$, with $\partial_{z} \mathscr{C}=\left|\partial_{z} \mathbf{U}\right|$ in (2). Averages over $\sigma$ bins with standard deviations are shown in Fig. 6 . The bin averages in both Fig. 6a and Fig. 6b, increase with $\sigma$ at about unity slope to breakpoints at $\sigma_{B m}=0.10$ and $\sigma_{B s}=0.07$, respectively. Deeper they become approximately constant at 

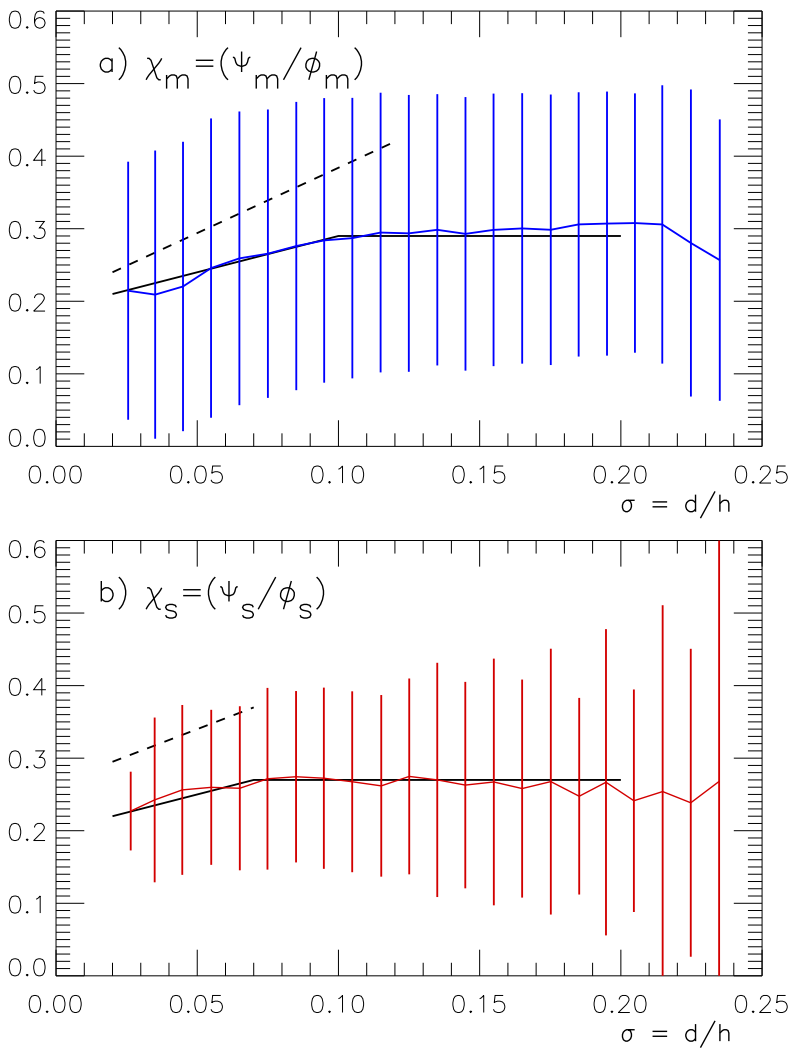

FIG. 6. Calculations of $\chi_{m}=\Psi_{m} \phi_{m}^{-1}$ and $\chi_{s}=\Psi_{s} \phi_{s}^{-1}$ across the surface layer to depths of $0.25 h$, with Stokes forcing, shown as averages over $\sigma$ bins of width 0.01 , with vertical bars extending \pm 1 standard deviation: (a) $\chi_{m}$ with $\zeta$ from (20) for $96 \mathrm{~h}$ (blue) from the regimes of Fig. 5 plus the low wind regime A23-31 and the transition regime A32-43, as well as D1-26 from D24S. (b) As in (a), but for $\chi_{s}$ (red) and excluding D24S. The dashed black lines are rough fits to the bin averages, with $\zeta$ from (1).

least to about $\sigma=0.20$. A similar exercise with $R=0$ gives significantly steeper slopes of 1.8 and 1.5 , respectively, as shown by the dashed lines. In addition to weakening depth dependencies, modifying $\zeta$ reduces the standard deviations in the range $0.08<\sigma<0.12$ from 0.33 to 0.20 for $\chi_{m}$ in Fig. 6a and from 0.15 to 0.13 for $\chi_{s}$ in Fig. 6b. Therefore, $\zeta$ will hereafter be defined by

$$
\zeta=\frac{d}{L}\left[1+\frac{P_{S}}{P_{U}} \mathrm{La}^{-2}\right]^{-1} .
$$

In No-Stokes boundary layers, the Monin-Obukhov depth $L$ is identified with the depth where the buoyant production, or suppression, of TKE equals the rate of shear production (Wyngaard 2010). Adding Stokes production to that of shear would increase this depth by a factor that (20) says is the term in square brackets.

An obvious effect of the Stokes forcing is to reduce the nondimensional gradients to less than one-half their
No-Stokes values, such that $\chi_{m}$ and $\chi_{s}$ fall from 1 to less than 0.5 as the Stokes forcing increases. Before examining this behavior, the vertical noise contribution to the spread is reduced by averaging $\chi_{m}$ and $\chi_{s}$ over four depth levels between $d_{o}$ (Table 2) and $\varepsilon h$. In general, more averaging does not reduce the spread further. The averaging requires that calculations at $\sigma$ less than the breakpoints be shifted to a reference depth, such as $\varepsilon$, according to the solid lines of Fig. 6:

$$
\begin{array}{cc}
\chi_{m}(\varepsilon)=\chi_{m}(\sigma)+1(\varepsilon-\sigma) ; & 0.02<\sigma<\sigma_{B m}, \\
\chi_{s}(\varepsilon)=\chi_{s}(\sigma)+1(\varepsilon-\sigma) ; & 0.02<\sigma<\sigma_{B s},
\end{array}
$$

where the factor 1 reflects the unity slopes.

A total of 273 hourly values of depth average $\chi_{m}(\varepsilon)$ and $\chi_{s}(\varepsilon)$ are computed from the data of Fig. 6 plus regimes A34-42 and A43-50 (excluding the day-night transition from hours 39 to 42 ), regime $\mathbf{J} 28-32$, as well as regime D41-100 of both D12S and D06S. Even with additional regimes, the overall standard deviation in $\chi_{m}(\varepsilon)$ is reduced from 0.20 (Fig. 6a) to only 0.082 , while in $\chi_{s}(\varepsilon)$ it is lowered from 0.13 (Fig. 6b) to 0.08 . These reductions are quantitative measures of the effectiveness of the depth averaging.

The independent variable, $V_{\text {Stokes }}$, is chosen to minimize the spread and maximize the correlation coefficients of $\chi_{m}$ and $\chi_{s}$ as functions of a Stokes parameter $\xi$. In (20), $\zeta$ is proportional to the ratio $\mu_{B}^{3} /\left(\mu_{U}^{3}+\mu_{S}^{3}\right)$, so by analogy an option for the Stokes parameter would be $\left(V_{\text {Stokes }} \mu^{-1}\right)^{3}=\mu_{S}^{3} /\left(\mu_{U}^{3}+\mu_{S}^{3}\right)$. A related second option that does not discriminate against buoyant production, or suppression is the fraction of total TKE production due to the presence of Stokes shear:

$$
\xi=\frac{\mu_{S}^{3}}{\left(\mu_{U}^{3}+\mu_{B}^{3}+\mu_{S}^{3}\right)} .
$$

Both $\chi_{m}(\varepsilon)$ and $\chi_{s}(\varepsilon)$ are plotted against this $\xi$ in Fig. 7. Quadratic regressions give the Stokes similarity functions:

$$
\begin{gathered}
\chi_{m}(\varepsilon)=1.05-2.43 \xi+1.69 \xi^{2}, \\
\chi_{s}(\varepsilon)=0.80-1.30 \xi+0.77 \xi^{2},
\end{gathered}
$$

with correlation coefficients greater than 0.89 . The standard deviation of departures of $\chi_{m}$ from the regression (24) is 0.035 , and that of $\chi_{s}$ from (25) is 0.031 . These further reductions of standard deviations by a factor of about 2, justifies the choice of $\xi$ from (23). However, with the first option above, the correlations are only slightly smaller (0.88 and 0.89$)$, and standard deviations marginally greater (0.037 and 0.032$)$. 

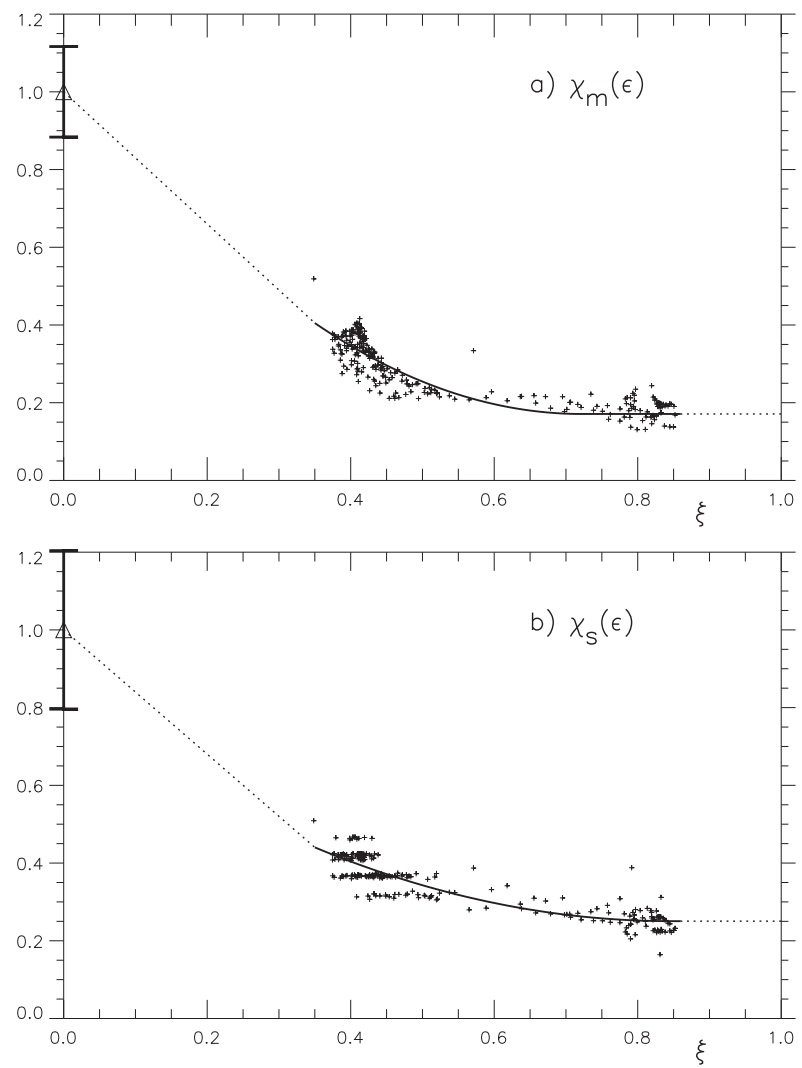

FIG. 7. Stokes similarity functions of $\xi$ (23) from $268 \mathrm{~h}$ of AprS, JunS, D24S, D12S, and D06S, excluding only regime A23-31, the first $12 \mathrm{~h}$ and regime J33-41 of JunS, as well as D1-26 and D27-40 of both D12S and D06S: (a) $\chi_{m}(\varepsilon)$, with the solid line following the quadratic regression from $\xi=0.35$ to its minimum at $\xi=0.72$ and then constant to the data limit at $\xi=0.86$, with the mean (1.00) and standard deviation from Fig. 5c shown at $\xi=0$. (b) As in (a), but for $\chi_{s}$ following the quadratic to $\xi=0.85$ and the mean and standard deviation at $\xi=0$ from Fig. 5d. The dotted traces are linear interpolations to 1 at $\xi=0$ and constant extrapolation beyond $\xi=0.86$.

Furthermore, the Eulerian shear $\left|\partial_{z} \mathbf{U}\right|$ will continue to be the choice for $\partial_{z} \mathscr{Q b}$ in (2), because using the Lagrangian shear nearly triples the standard deviation, and $\chi_{m}(\varepsilon)$ becomes a nearly linear increasing function of $\xi$, with an intercept at about 0.3 .

A smooth transition to No-Stokes forcing requires the intercepts to be $\chi_{m}=\chi_{s}=1$ at $\xi=0$. Indeed both curves do trend toward these points (triangles with \pm 1 standard deviation error bars from Fig. 5). However, until the data gaps are filled a reasonable approach is to use the solid lines of (24) plus the dotted linear interpolations for $\xi$ between 0.0 and 0.35 and constant extrapolation at the minimum values of quadratics at $\xi=0.72$ for $\chi_{m}$ and $\xi=0.85$ for $\chi_{s}$. The respective constants are about 0.17 and 0.25 , and their reciprocals, 5.8 and 4.0 , are the maximum factors that the turbulent velocity scales, $w_{m}$ of (13) and $w_{s}$ of (14), can be enhanced by Stokes forcing. The ratio of these factors, 1.46 , becomes the upper limit of the Prandtl number.

\section{Parameterizing the ocean surface layer}

Use of the Stokes similarity functions (24) and (25) in OGCMs, requires knowledge of $\mu_{U}^{3}, \mu_{S}^{3}$, and $\mu_{B}^{3}$, as well as of the meteorological and Stokes forcing. Given this forcing and a diagnosed boundary layer depth, it is straightforward to compute $u^{* 3}, \mathrm{La}^{-2}$, and $w^{* 3}$, so the problem reduces to finding parameterizations for $P_{U}$, $P_{S}$, and $P_{B}$, as computed in section $4 \mathrm{~b}$. The regimes of Fig. 7 are used again for this purpose.

First consider $\mu_{B}^{* 3}=P_{B} w^{* 3}$ from (12). Assuming a linear buoyancy flux profile from the surface to zero at a depth $h_{0}$ gives $P_{B}=0.095$ for $h_{0}=h$, so $P_{B}$ may be well represented by a somewhat smaller constant, if the ratio $h_{0} / h<1$ is relatively constant. Indeed, $227 \mathrm{~h}$ where the buoyancy production is more the $1 \%$ of the total, give an average of 0.090 with a standard deviation of 0.006 . Thus, the parameterization $P_{B}^{\mathrm{PAR}}=0.090$ is good to within $7 \%$ when it matters.

The parameterization of $\mu_{S}^{3}=P_{S} u^{* 3} \mathrm{La}^{-2}$ from (11) becomes the numerator of $\xi$ in (23) and a significant term in the denominator. The agreement between the above linear profile assumption and $P_{B}$, suggests making a similar assumption about the momentum flux profile. A linear profile in (11) gives an estimate:

$$
P_{S e}=\left(\rho u^{* 3} \mathrm{La}^{-2}\right)^{-1} \int\left(1+\frac{z}{h}\right)\left[\boldsymbol{\tau} \cdot \partial_{z} \mathbf{U}_{S}\right] d z
$$

Figure 8 compares 290 LES calculations of $P_{S}$ to these estimates with comparable integration limits (first model level below $\varepsilon h$ up to $-\Delta Z_{\min } / 2$ ) from section $4 \mathrm{~b}$. The linear regression (dashed line) gives a correlation coefficient higher than 0.99 , but does not go through the origin. A compromise with the best fit through the origin gives the parameterization, $P_{S}^{\mathrm{PAR}}=0.94 P_{S e}$, which lies within $5 \%$ of the linear regression over the range of Fig. 8.

Finally consider $\mu_{U}^{3}=P_{U} u^{* 3}$ and its distinctly smaller values with Stokes forcing (AprS and JunS) than without. From (2) and (4), the shear in the integrand of (10) should scale with $u^{*} \phi_{m} \chi_{m}$, rather than just $u^{*}$, but competing effects complicate the momentum flux. The success of (20) in reducing depth dependencies in section $4 \mathrm{~b}$, suggests scaling it with $\mu^{2}$, rather than $u^{* 2}$. Additional scaling by $\chi_{m}^{-1}$, to account for enhancement of this flux from Langmuir turbulence, leads to a dependence of $P_{U}$ on a combined parameter, $\Lambda=\phi_{m}\left(1+\mathrm{La}^{-2} P_{S} / P_{U}\right)$. The close fit of the LES data 


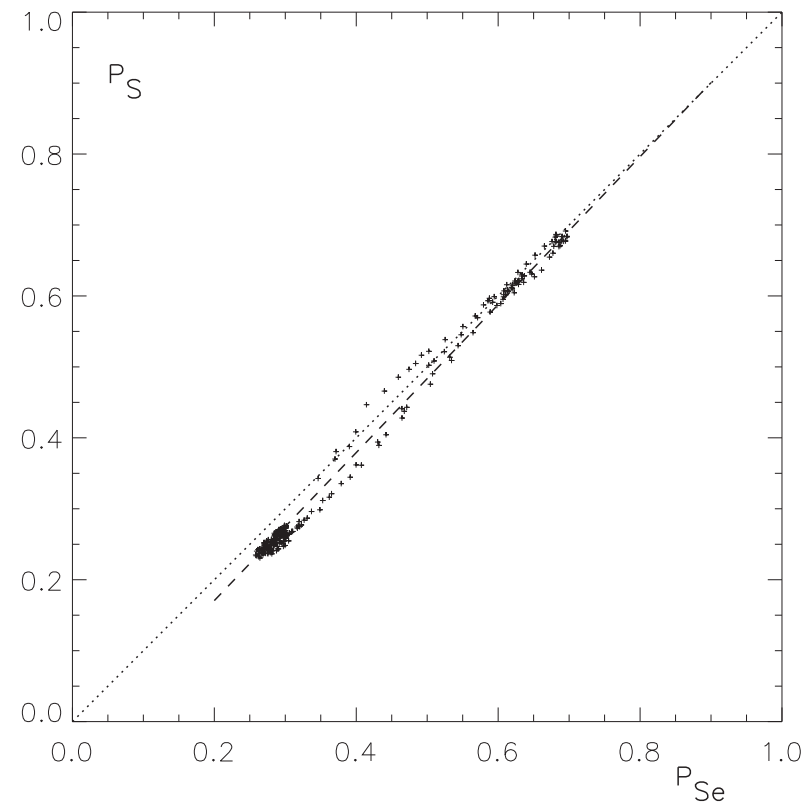

FIG. 8. The Stokes shear TKE production parameter $P_{S}$ computed from the periods of Fig. 7 plus hours 5-12 and regime J33-41 of JunS, with (11) compared to the estimate $P_{S e}$ from (26), with the same integration limits (see text). A linear regression gives the dashed line and a correlation coefficient of 0.99 .

in Fig. 9a supports the above scaling and the cancellation of $\chi_{m}$, in particular. The nonlinear behavior complicates the parameterization of $P_{U}$, but a simple solution is to relate $P_{U}$ directly to $\Lambda^{-1}$, as shown in Fig. 9b. The intercept, slope, and correlation coefficient given by the linear regression (dashed) are $b_{\Lambda}=0.91$ and $m_{\Lambda}=3.60$ and 0.98 , respectively. The linear fit leads to a quadratic equation in $P_{U}$, and the solution gives the parameterization

$$
\begin{aligned}
P_{U}^{\mathrm{PAR}} & =\left(f_{\Lambda}^{2}+b_{\Lambda} \mathrm{La}^{-2} P_{S}^{\mathrm{PAR}}\right)^{1 / 2}-f_{\Lambda} ; \\
f_{\Lambda} & =\frac{1}{2}\left(\mathrm{La}^{-2} P_{S}^{\mathrm{PAR}}-b_{\Lambda}-\frac{m_{\Lambda}}{\phi_{m}}\right) .
\end{aligned}
$$

Since $\phi_{m}(\varepsilon)$ in (27) with $\zeta$ from (20) requires $P_{U}^{\mathrm{PAR}}$, it is necessary to iterate. Starting from an initial $P_{U}^{\mathrm{PAR}}=2.5$, as suggested by Fig. 9, one iteration is sufficient.

The benefit of incorporating buoyancy forcing and $\mu^{2}$ from section $4 \mathrm{~b}$ in the scaling of (10) is demonstrated by setting $\phi_{m}=1$ (neutral) and $P_{S}=P_{U}$, then plotting $P_{U}$ against the resulting function of Langmuir number only, $\left(1+\mathrm{La}^{-2}\right)^{-1}$, in Fig. 9c. Not only does the correlation fall to 0.67 , but very few points fall near the linear regression (dashed). Also, the dark blue triangles from the stable forcing of regime A32-43, and the light blue diamonds from the near neutral regime A44-50 all cluster below the other points. Overall, the major
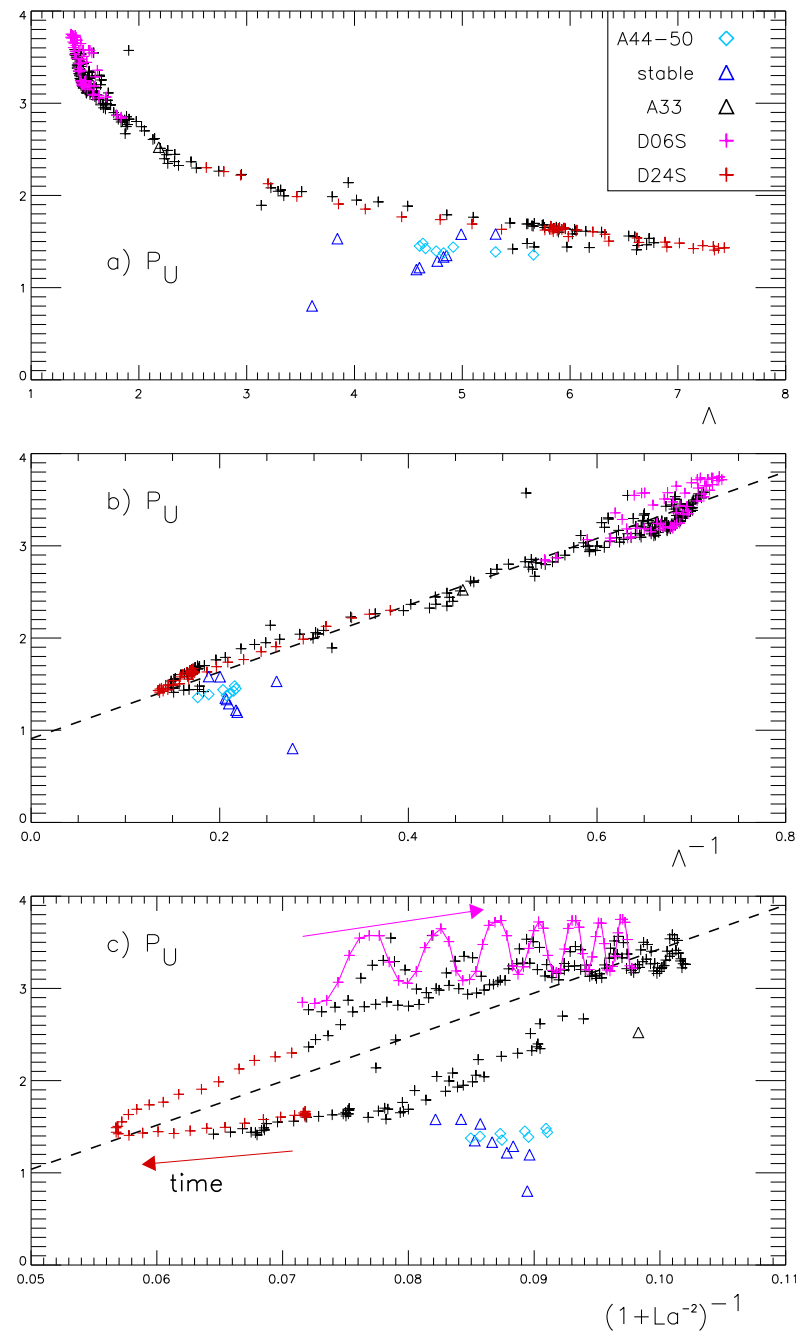

FIG. 9. Parameterizing the mechanical surface layer TKE production parameter $P_{U}$ from $290 \mathrm{~h}$ over the periods of Fig. 8: (a) vs $\Lambda=\phi_{m}\left(1+\mathrm{La}^{-2} P_{S} / P_{U}\right) ;(\mathrm{b})$ vs $\Lambda^{-1}$; and (c) vs $\left(1+\mathrm{La}^{-2}\right)$. The red points are from $\mathrm{D} 24 \mathrm{~S}$; regime $\mathrm{D} 1-26$, with the time progression in (c) indicated by the red arrow, and from regime D27-40. The magenta points are from regime D41-100 of D06S, with the arrow in (c) indicating their time progression. The triangles are from the transition regime A32-43, with dark blue indicating stable buoyancy forcing. The light blue diamonds span the near-neutral regime A44-50.

effect of $P_{S}^{\mathrm{PAR}}$ in $\Lambda$ is to move red, blue, and black points below the regression line of Fig. $9 \mathrm{c}$ far to the left in Fig. 9b, relative to points with higher $P_{U}$, and to move points (e.g., the magenta) with high $P_{U}$ to the right. The blue points remain clustered, because as the boundary layer deepens and during the day-night transition (Fig. 3c), the decrease in $\phi_{m}$ from greater than 1 in stable forcing to less than 1 , is compensated by an increase in $\left(P_{S} / P_{U}\right)$ at about the same $\mathrm{La}^{-2}$.

Multiple values of $P_{U}$ are a problem for any attempt to parameterize $P_{U}$ in terms of $\mathrm{La}^{-2}$ alone. For example, 
consider the red points from D24S in Fig. 9c. After $\mathrm{La}^{-2}$ rises after hour 12 (red time arrow) then falls to hour 39 (Fig. 1d), $P_{U}$ is about $50 \%$ higher (2.4) at $(1+$ $\left.\mathrm{La}^{-2}\right)^{-1}=0.72$, than initially (1.6). However, this behavior is not evident Fig. 9b, because as $\mathrm{La}^{-2}$ increases, $P_{S}$ falls, such that with $P_{U}$ relatively steady, so is $\Lambda^{-1}$ at about 0.11 . Then later, as winds and waves diminish, both $\mathrm{La}^{-2}$ and $P_{S}$ decrease, such that $P_{U}$ increases nearly linearly with $\Lambda^{-1}$ and becomes a factor of 1.5 higher than earlier for the same $\mathrm{La}^{-2}$.

Large oscillations are evident in the points of Fig. 9c from D06S (magenta) as time advances after hour 41 (magenta arrow) and the amplitude of the inertial motions is about $12 \mathrm{~cm} \mathrm{~s}^{-1}$. However, there are about seven cycles in $58 \mathrm{~h}$ (solid magenta curve), so the period is only about half the inertial, because the coupling of the inertial current to the wind stress weakens with depth. Twice every inertial period the dot product of (10), and hence $P_{U}$, are minima, because the current is nearly orthogonal to the wind stress. When the current then rotates either more downwind, or upwind, the wind stress accelerates the upper flow in the direction of the wind sooner than the deeper. Therefore, with either rotation a downwind shear develops that increases the dot product, and hence both $P_{U}$ and $\Lambda^{-1}$, until they are all maxima when the current is near downwind, or upwind. In Fig. 9b, these oscillations have a relatively narrow range from about 0.55 to 0.73 in $\Lambda^{-1}$ and are in phase with $P_{U}$, such that the magenta points now scatter about the regression line. Another contributor to the narrow range and reduced scatter is inertial variability in $h$ that affects both $\phi_{m}(\varepsilon)$ and the integration limits of (10).

\section{a. Evaluating the parameterization}

A full parameterization has been developed for the velocity scales associated with turbulent viscosity $w_{m}^{\mathrm{PAR}}$ and turbulent diffusivity $w_{s}^{\mathrm{PAR}}$ in terms of the meteorological forcing and Stokes drift profile, given the boundary layer depth. It is summarized as follows:

- $P_{B}^{\mathrm{PAR}}=0.090$

- $P_{S}^{\mathrm{PAR}}=0.94 P_{S e}$, with $P_{S e}$ from (26)

- initial $P_{U}^{\mathrm{PAR}}=2.5$

- $\phi_{m}(\varepsilon)$ from (18), with $\zeta$ at $d=\varepsilon h$, from (20) using $P_{S}^{\text {PAR }}$ and $P_{U}^{\mathrm{PAR}}$

- update $P_{U}^{\mathrm{PAR}}$ from (27), using $P_{S}^{\mathrm{PAR}}$ and $\phi_{m}(\varepsilon)$

- $\xi$ from (23), using $P_{U}^{\mathrm{PAR}}$ in (10), $P_{S}^{\mathrm{PAR}}$ in (11) and $P_{B}^{\mathrm{PAR}}$ in $(12)$

- $\chi_{m}(\xi)$ from (24) and $\chi_{s}(\xi)$ from (25);

- $\phi_{m}(\varepsilon)$ from (18), and $\phi_{s}(\varepsilon)$ from (19), using $P_{S}^{\text {PAR }}$ and updated $P_{U}^{\mathrm{PAR}}$ in (20)
- $w_{m}^{\text {PAR }}$ from (13), using $\chi_{m}(\xi)$ and $\phi_{m}(\varepsilon)$

- $w_{s}^{\text {PAR }}$ from (14), using $\chi_{s}(\xi)$ and $\phi_{s}(\varepsilon)$.

To evaluate the parameterization, the turbulent velocity scales are calculated from the LES fluxes within the surface layer. These scales are then referenced to $\sigma=\varepsilon h$ to be comparable to the parameterized values:

$$
\begin{aligned}
& w_{m}^{\mathrm{LES}}(\varepsilon)=w_{m}(\sigma) \frac{\phi_{m}(\sigma)}{\phi_{m}(\varepsilon)} \frac{\chi_{m}(\sigma)}{\chi_{m}(\varepsilon)} ; \quad w_{m}(\sigma)=\frac{\left|\left\langle w^{\prime} \mathbf{u}^{\prime}\right\rangle\right|}{d\left|\partial_{z} \mathbf{U}\right|} \\
& w_{s}^{\mathrm{LES}}(\varepsilon)=w_{s}(\sigma) \frac{\phi_{s}(\sigma)}{\phi_{s}(\varepsilon)} \frac{\chi_{s}(\sigma)}{\chi_{s}(\varepsilon)} ; \quad w_{s}(\sigma)=\frac{-\left\langle w^{\prime} \theta^{\prime}\right\rangle}{d \partial_{z} \Theta}
\end{aligned}
$$

The evaluation is as stringent as practical. It uses LES turbulent fluxes that are not part of the parameterization development, rather than the surface fluxes that are. It includes $275 \mathrm{~h}: 87 \mathrm{~h}$ from AprN and JunN, $88 \mathrm{~h}$ from AprS and JunS, plus all $100 \mathrm{~h}$ of D06S. Thus, the parameterization is challenged by three regimes not used in its development; the weak and variable winds of A23-31 as well as regimes D1-26 and D27-40 of D06S when the wind decay is most rapid and $\mathrm{La}^{-2}$ reaches its maximum. All regimes from D24S and D12S are excluded because they are similar to D06S.

The calculations of $\chi_{m}$ and $\chi_{s}$ for use in (28) and (29) are subject to similar depth averaging constraints (four consecutive depths between $d_{o}$ and $\varepsilon h$ ), as discussed in section 4 . Since the linear depth dependence of (13) and (14) is more valid nearer the surface the averaging stays as near as possible to $d_{o}$ and varies with regime according to Table 2 . An exception is the strongwind, weak-buoyancy-forced regime D1-26, where the LES solutions suggest $d_{o}=0.3 \mathrm{~m}$ and no depth averaging of (29) over the first $16 \mathrm{~h}$.

Parameterized turbulent velocity scales are compared to the LES in Fig. 10. For both $w_{m}$ and $w_{s}$ the correlation coefficient is 0.97 , but there is a small high bias, especially for $w_{m}^{\mathrm{PAR}}$. This bias suggests a less rapid increase with depth than linear, which would effectively decrease " $d$ " in the denominators of (28) and (29) and increase the LES values, especially the smaller when the forcing is weaker. There is relatively little scatter in AprN and JunN (blue points), which are confined to values less than $1.5 \mathrm{~cm} \mathrm{~s}^{-1}$. Also in this region are the D06S points (red) from regime D41100. The clusters of red D06S points near $w_{m}^{\mathrm{LES}}=$ $6.5 \mathrm{~cm} \mathrm{~s}^{-1}$, and $w_{s}^{\text {LES }}=3.5 \mathrm{~cm} \mathrm{~s}^{-1}$ come from regime D1-26, then during D27-40 there is a relatively smooth transition of the red D06S points while $\mathrm{La}^{-2}$ falls from its peak. Wind speed and direction and the Stokes 

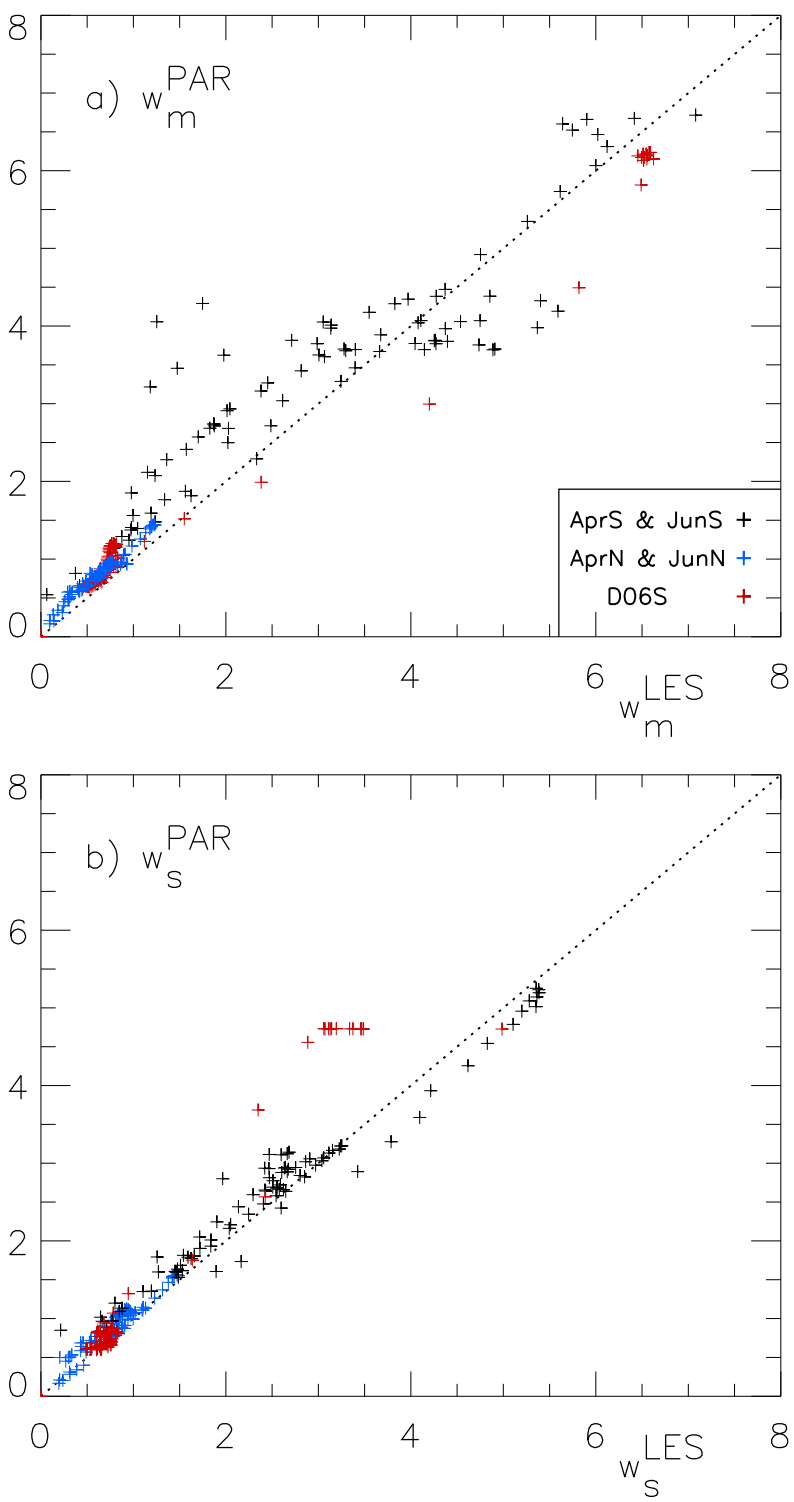

FIG. 10. Turbulent velocity scales $\left(\mathrm{cm} \mathrm{s}^{-1}\right)$ from the AprN and JunN No-Stokes LES (87 blue points), from the AprS and JunS Stokes LES (88 black points), and from all of D06S (100 red points): a) parameterized $w_{m}^{\mathrm{PAR}}$ vs $w_{m}^{\mathrm{LES}}$ computed in the surface layer (depths between $d_{o}$ and $<\varepsilon h$ ); and (b) parameterized $w_{s}^{\text {PAR }}$ vs $w_{s}^{\text {LES }}$

drift all vary only in AprS and JunS (black points). This combined variability tends to produce more spread; more so for $w_{m}$ (Fig. 10a) than for $w_{s}$ (Fig. 10b).

This qualitative comparison of Fig. 10 and Table 3 indicates that overall the LES turbulent velocity scales at $\sigma=\varepsilon$ are well represented by $w_{m}^{\mathrm{PAR}}$ and $w_{s}^{\mathrm{PAR}}$ throughout the whole of the transient forcing of Fig. 1, but with obvious outliers, periods of systematic bias and some sensitivity to the depth averaging. However, the general behavior of the Prandtl number in (15) is robust with respect to all these issues. For AprN and JunN (blue points) it is less than 1 , but with sufficient Stokes forcing it becomes greater than 1 . With the Stokes similarity functions (24) and (25) from (Fig. 7) giving $\chi_{m}<\chi_{s}$, this behavior is captured by the parameterization.

\section{Exploring simpler alternatives}

The parameterization of section 5 assumes given Stokes profiles, but these are not always available. To demonstrate the benefits of a detailed calculation, for example (17), two simpler possibilities are considered. In the first the Langmuir number is the only wave information available, and in the second there is no knowledge of the wave field. Comparisons with $w_{m}^{\mathrm{LES}}$ and $w_{s}^{\mathrm{LES}}$ at $\varepsilon$ from a total of $188 \mathrm{~h}$ from AprS, JunS, and D06S are shown in Fig. 11. The statistics are summarized Table 3, along those of $w_{m}^{\mathrm{PAR}}$ and $w_{s}^{\mathrm{PAR}}$ from the same times.

Previously, Stokes LES have been used to develop alternative scalings that depend on Langmuir number, but not the Stokes drift profile. For example, McWilliams and Sullivan (2000) express the turbulent viscosity and diffusivity velocities, respectively, as

$$
\begin{aligned}
w_{m}^{\mathrm{MS}} & =\frac{\kappa u^{*}}{\phi_{m}(\zeta)} \mathscr{E} ; \quad w_{s}^{\mathrm{MS}}=\frac{\kappa u^{*}}{\phi_{s}(\zeta)} \mathscr{E} ; \\
\mathscr{E} & =\left(1+A \mathrm{La}^{-4}\right)^{1 / 2} ; \quad A=0.080,
\end{aligned}
$$

using the functions (6), (7), and (8), and where $\varepsilon$ is the enhancement due to Stokes effects. The most notable feature of Fig. 11a is that $w_{m}^{\mathrm{MS}}$ tends to be bias high at small values and low at larger, such that the slope of the regression (dashed) is only about 0.71 . In particular, the high values of $w_{m}^{\mathrm{LES}}>5 \mathrm{~cm} \mathrm{~s}^{-1}$ are not reproduced and the rms difference is 0.91 compared to $0.58 \mathrm{~cm} \mathrm{~s}^{-1}$ for $w_{m}^{\mathrm{PAR}}$ (Table 3). There is a significant high bias and considerable scatter of $w_{s}^{\mathrm{MS}}$ in Fig. $11 \mathrm{~b}$ for $0.5<w_{s}^{\mathrm{LES}}<$ $3 \mathrm{~cm} \mathrm{~s}^{-1}$, which leads to an rms difference about thrice that of $w_{s}^{\mathrm{PAR}}$ and to the highest mean bias, 1.47.

Perhaps to address such biases and scatter, Smyth et al. (2002) retain the form of (30), but add a dependency on $w^{* 3}$, and hence $\mu_{B}^{3}$, through

$$
A=0.15\left(\frac{u^{* 3}}{u^{* 3}+0.6 w^{* 3}}\right)^{2} .
$$

The resulting estimates, with $w^{*}=0$ in stable conditions, are denoted as $w_{m}^{\mathrm{SS}}$ and $w_{s}^{\mathrm{SS}}$. The latter is seen in Fig. 11d to be an improvement over $w_{s}^{\mathrm{MS}}$ (Table 3). The rms difference is reduced to 0.91 and the high mean bias to 1.06. In Fig. $11 \mathrm{c}$ there is an improvement of $w_{s}^{\mathrm{SS}}$ over $w_{s}^{\mathrm{MS}}$ 
TABLE 3. Summary statistics from comparisons of turbulent velocity scales with Stokes forcing from $188 \mathrm{~h}$ of AprS, JunS, and D06S. These scales $\left(X=\left\{w_{m}^{\mathrm{LES}} ; w_{s}^{\mathrm{LES}}\right\}\right)$ are compared to the parameterization of section 5 with computed Stokes $\operatorname{drift}\left(Y=\left\{w_{m}^{\mathrm{PAR}} ; w_{s}^{\mathrm{PAR}}\right\}\right)$, and with bulk Stokes drift $\left(Y=\left\{w_{m}^{\mathrm{BSD}} ; w_{s}^{\mathrm{BSD}}\right\}\right)$. Other alternatives from section 6 are $\left(Y=\left\{w_{m}^{\mathrm{MS}} ; w_{s}^{\mathrm{MS}}\right\}\right)$ from McWilliams and Sullivan $(2000)$, and $\left(Y=\left\{w_{m}^{\text {ss }} ; w_{s}^{\text {ss }}\right\}\right)$ from Smyth et al. (2002). The standard deviations are $S_{y}$ and $S_{x}$, rms is the root-mean-square difference, the correlation coefficient is $r_{x y}$, and the mean bias $\bar{Y} / \bar{X}$ gives the slope of the best least squares fit through the origin.

\begin{tabular}{|c|c|c|c|c|c|c|c|c|}
\hline Figure & $Y$ & $\bar{Y}\left(\mathrm{~cm} \mathrm{~s}^{-1}\right)$ & $\bar{X}\left(\mathrm{~cm} \mathrm{~s}^{-1}\right)$ & $S_{y}\left(\mathrm{~cm} \mathrm{~s}^{-1}\right)$ & $S_{x}\left(\mathrm{~cm} \mathrm{~s}^{-1}\right)$ & $\operatorname{rms}\left(\mathrm{cm} \mathrm{s}^{-1}\right)$ & $r_{x y}$ & $\bar{Y} / \bar{X}$ \\
\hline Fig. 10a & $w_{m}^{\mathrm{PAR}}$ & 2.47 & 2.26 & 1.90 & 2.03 & 0.58 & 0.97 & 1.09 \\
\hline Fig. 11a & $w_{m}^{\mathrm{MS}}$ & 1.99 & 2.26 & 1.36 & 2.03 & 0.91 & 0.96 & 0.88 \\
\hline Fig. 11c & $w_{m}^{\mathrm{sS}}$ & 1.59 & 2.26 & 1.64 & 2.03 & 1.14 & 0.92 & 0.70 \\
\hline Fig. 11e & $w_{m}^{\mathrm{mSD}}$ & 2.28 & 2.26 & 1.66 & 2.03 & 0.78 & 0.95 & 1.00 \\
\hline Fig. 10b & $w_{s}^{m}$ & 1.92 & 1.75 & 1.43 & 1.32 & 0.44 & 0.96 & 1.10 \\
\hline Fig. 11b & $w_{s}^{\mathrm{MS}}$ & 2.58 & 1.75 & 1.73 & 1.32 & 1.31 & 0.89 & 1.47 \\
\hline Fig. 11d & $w_{s}^{S S S}$ & 1.85 & 1.75 & 1.63 & 1.32 & 0.91 & 0.89 & 1.06 \\
\hline Fig. 11f & $w_{s}^{\mathrm{BSD}}$ & 1.80 & 1.75 & 1.16 & 1.32 & 0.29 & 0.98 & 1.03 \\
\hline
\end{tabular}

low values, but not at high. The rms difference is even bigger (1.14) and the overall mean even smaller (1.59 versus $\left.1.99 \mathrm{~cm} \mathrm{~s}^{-1}\right)$, such that the mean bias $(0.70)$ becomes the lowest in Table 3.

Thus, by these measures, the formulation of (30) with either (31) or (32) is not as representative of the LES velocity scales as the parameterization of section 5 . In particular, because the viscosity and diffusivity only differ by the ratio of $\phi_{s}$ to $\phi_{m}$, the Prandtl number (15) never exceeds unity regardless of Stokes effects, in contrast to the LES with $\operatorname{Pr}>1$.

The approach to the second situation of no wave information is to apply the section 5 parameterization with the following bulk Stokes drift (BSD) approximation of the profile, given by the meteorological forcing alone. This profile is discretized to be consistent with (17) and similarly used in (26) to give to give a bulk estimate $P_{S}^{\mathrm{BSD}}$ and hence Stokes surface layer production, $\mu_{S}^{3}=P_{S}^{\mathrm{BSD}} u^{* 3} \mathrm{La}^{-2}$ in (23). The assumptions are wind-wave alignment and equilibrium, $\mathrm{La}^{-2}=11$, as well as a monochromatic wave where the profile is exponential:

$$
\left|\mathbf{U}_{S}(z)\right|=u^{*} \mathrm{La}^{-2} e^{-\sigma / \eta},
$$

and the vertical decay scale is $\eta=(2 k h)^{-1}$, for a horizontal wavenumber $k$. Then, following Alves et al. (2003) 1.2 approximates the wave age (the ratio of phase speed $c_{p}$ to wind speed), so that $\left(c_{p} / 1.2\right)^{2}=\left(855 C_{D}^{-1} u^{* 2}\right)$, where 855 is the ratio of ocean to air density, and $C_{D}$ is the drag coefficient. Finally, assume linear dispersion to give $k=g c_{p}^{-2}$, and hence $\eta$ proportional to $u^{* 2} /(g h)$. The resulting turbulent velocity scales, $w_{m}^{\mathrm{BSD}}$ and $w_{s}^{\mathrm{BSD}}$, are compared to LES values in Figs. 11e and 11f, respectively.

With (33), the integral (26) can also be computed analytically from $z=-\varepsilon h$ to the surface:

$$
P_{S}^{\mathrm{ASD}}=1-\eta-(1-\eta-\varepsilon) e^{-\varepsilon / \eta},
$$

which is bounded by $\varepsilon$ at large $\eta$ and by 1 at $\eta=0$. This relation is shown by the black curve in Fig. 12, with $\eta$ the ordinate and $P_{S}^{\mathrm{ASD}}$ the abscissa. Figure 12 also shows $P_{S}$ from the LES versus $P_{S}^{\mathrm{ASD}}$. The radical departure of this comparison to that of the same $P_{S}$ to $P_{S e}$ from (26) in Fig. 8 is far more than expected from the discretization and different integration limits. It demonstrates that neither $P_{S}^{\mathrm{ASD}}$, nor $P_{S}^{\mathrm{BSD}}$ (not shown) give a consistent representation of $\mu_{S}^{* 3}$, across varying conditions.

At hour 19 of JunS (magenta diamond), for example, $\eta$ is about 0.04 , such that the black curve of 12 gives $P_{S}^{\mathrm{ASD}}=0.90$ (black diamond). This estimate is considerably higher than $P_{S}=0.60$, because $\eta$ fails to capture the rotation with depth of $\mathbf{U}_{S}$ away from the wind direction, as seen in Fig. 2b. This veering is caused by low-frequency swell propagating to the east-northeast and a growing wind sea to the southeast (Fig. 2a). During the progression in time (magenta arrow) to hour 31 (magenta triangle) the decay scale increases with the wind speed to about 0.065 , and hence $P_{S}^{\mathrm{ASD}}$ is reduced to about 0.77 (black triangle). The developing wind waves tend to align the near surface Stokes shear more with the wind (Fig. 2d) and $P_{S}$ increases. Thus, there is a fortuitous improvement in the agreement.

The circumstances are different at hour 1 of AprS (blue diamond). The severe underestimate of $P_{S}^{\mathrm{ASD}}=$ 0.45 is due to the very deep penetration of the Stokes drift ( $\eta=0.16$ from the black curve of Fig. 12), which with $h=180 \mathrm{~m}$ is still $60 \%$ of the surface drift at $\varepsilon h$. In contrast, the computed Stokes drift (not shown) at $\varepsilon h$ has fallen to less than $10 \%$, and the greater Stokes shear above makes $P_{S}$ relatively larger. Over the next several hours as indicated by the blue arrow, the low-frequency swell continues to dominate, so 

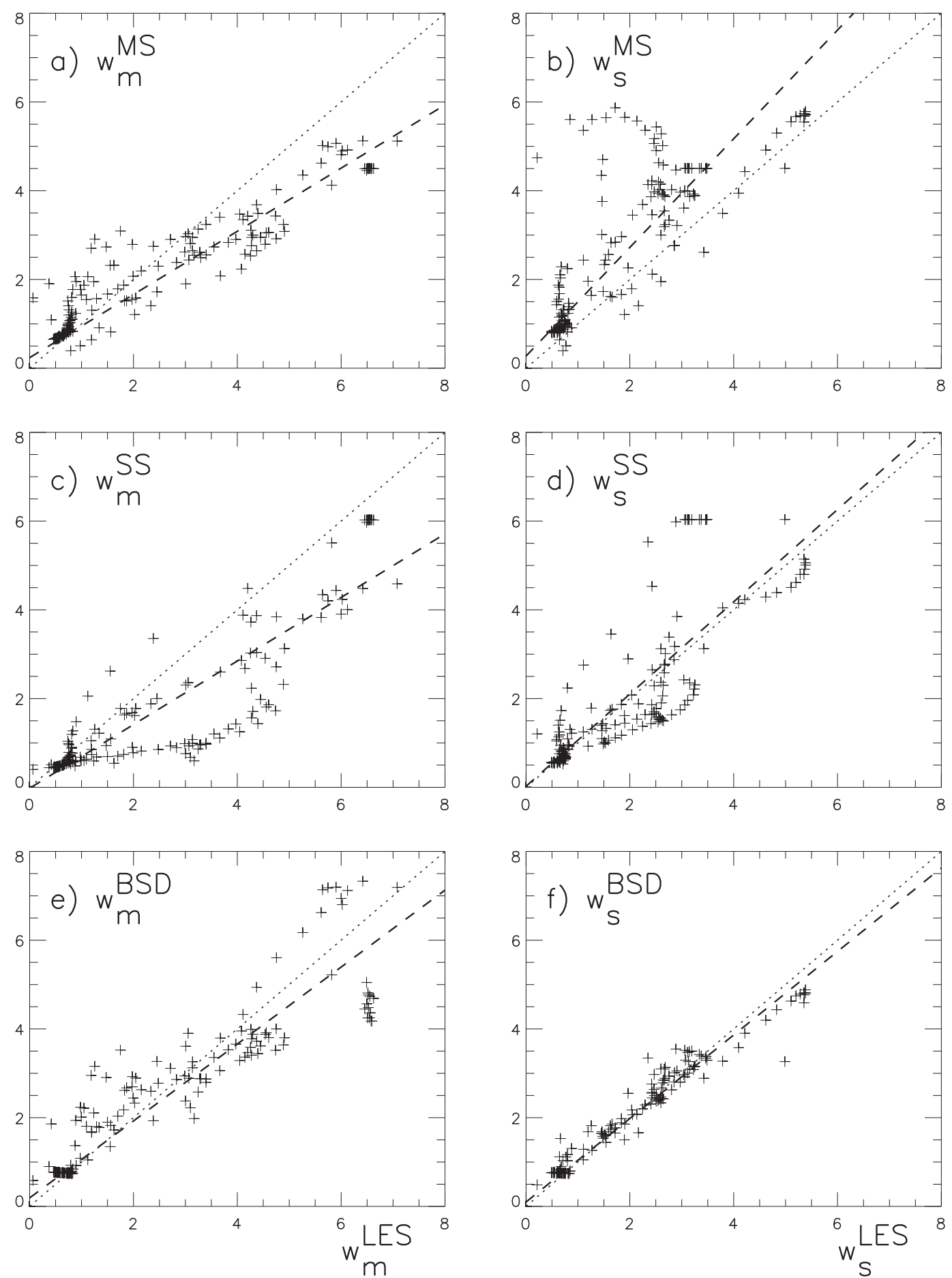

FIG. 11. Comparison of turbulent velocity scales $w_{m}^{\mathrm{LES}}$ and $w_{s}^{\mathrm{LES}}\left(\mathrm{cm} \mathrm{s}^{-1}\right)$ from $188 \mathrm{~h}$ of the Stokes AprS, JunS, and D06S LES, respectively with: (a) $w_{m}^{\mathrm{MS}}$ and (b) $w_{s}^{\mathrm{MS}}$, according to the scalings of McWilliams and Sullivan (2000); (c) $w_{m}^{\mathrm{SS}}$ and (d) $w_{s}^{\mathrm{SS}}$, following Smyth et al. (2002); (e) $w_{m}^{\mathrm{BSD}}$ and (f) $w_{s}^{\mathrm{BSD}}$, from the parameterization of section 5, but with discretized bulk Stokes drift. 


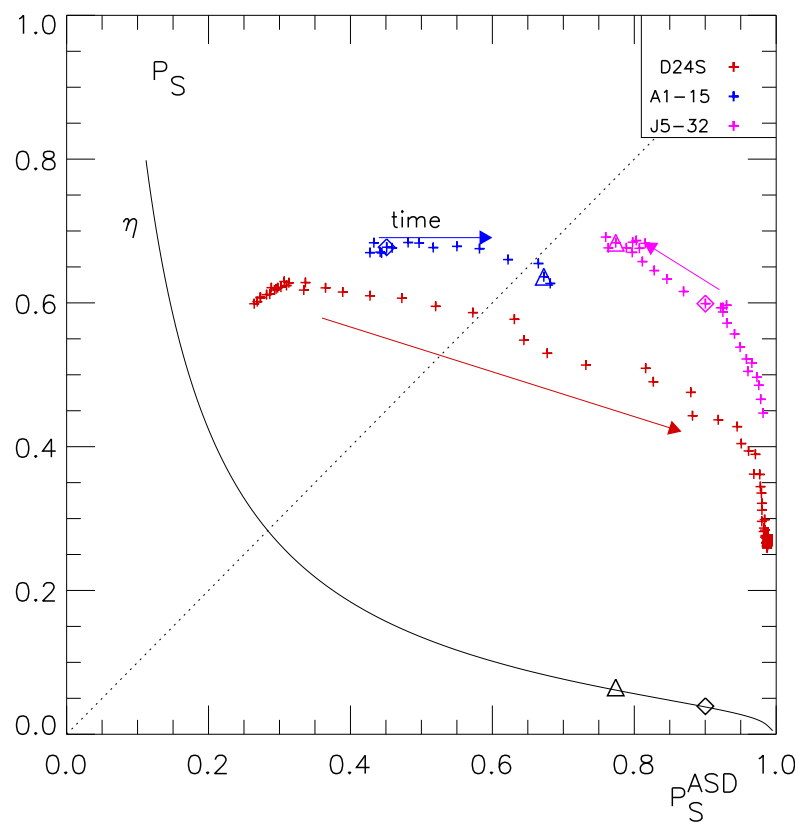

FIG. 12. As in Fig. 8, but comparing $P_{S}$ from the LES to the analytic $P_{S}^{\mathrm{ASD}}$ from (34) over: all of D24S (red); the first two regimes (A1-11, A12-15) of AprS (dark blue), with a diamond at hour 1 and triangle at hour 13 (local noon); and the J5-27 and J2832 regimes of JunS (magenta), with a diamond at hour 19 and triangle at hour 31 (local noon), corresponding to the waves of Fig. 2. Also shown for reference is the black curve from (34) that relates $\eta$ (now the ordinate) to $P_{S}^{\mathrm{ASD}}$ (still the abscissa). The black diamond and triangle give the values of $\eta$ at hours 19 and 31 of JunS, respectively.

$P_{S}$ stays relatively constant, but by hour 13 (blue triangle) the lower winds halve the decay scale to about 0.08 , which increases $P_{S}^{\mathrm{ASD}}$, such that the agreement again improves by chance.

Despite aligned wind and waves, $P_{S}^{\mathrm{ASD}}$ from D24S bears no relationship to $P_{S}$ (red points of Fig. 12). There is a continuous increase in $P_{S}^{\mathrm{ASD}}$ as $\eta$ decreases, initially because $h$ deepens, then because the wind decreases. In contrast, $P_{S}$ steadily decreases over D27-40 and D41-100.

The very poor representation of $P_{S}$, by $P_{S}^{\mathrm{ASD}}$ and $P_{S}^{\mathrm{BSD}}$ is not apparent in either Fig. 11e or Fig. 11f. Arguably, $w_{m}^{\mathrm{BSD}}$ and especially $w_{s}^{\mathrm{BSD}}$ are better representations of the LES than the other examples in Fig. 11. In particular, the Prandtl number does exceeds unity, because these calculations use the Stokes similarity functions, $\chi_{m}$ from (24) and $\chi_{s}$ from (25). Also, the rms differences are smaller, the means' biases are closer to unity, and the correlation coefficients are mostly greater (Table 3 ).

By these measures $w_{m}^{\mathrm{PAR}}$ appears to represent the LES somewhat better than $w_{m}^{\mathrm{BSD}}$, while the opposite holds for $w_{s}^{\mathrm{PAR}}$ compared to $w_{s}^{\mathrm{BSD}}$. However, the success of such a simple BSD would likely degrade when the Stokes forcing is less prominent than in the Southern
Ocean for two reasons: first, there would be less compensation of $P_{S}^{\mathrm{BSD}}$ errors in the numerator of $\xi$ from $P_{S}^{\mathrm{BSD}}$ in the denominator; and second, there would be more sensitivity of $\chi_{m}$ and $\chi_{s}$ to uncompensated errors at smaller values of $\xi$, as shown by Fig. 7 .

\section{Discussion and conclusions}

The Stokes similarity functions (24) and (25) are the key new LES results that should be represented in simpler models and parameterizations. They account for surface wave effects in the velocity scales for turbulent viscosity and diffusivity in the surface layer, across a wide range of highly variable forcing, with misaligned wind and waves. However, it is important to acknowledge the caveat that the results presented are valid only for the surface layer of the seven LES of our study, with $\xi$ equal zero and between about 0.35 and 0.86 . Unfortunately, available observations are insufficient to determine qualitatively how well these LES results apply to the real ocean, where other effects, such as wave breaking, may provide additional production of TKE that needs to be included in (23). However, the ARGO comparison of Fig. 3b is encouraging.

The Wyngaard (1982) view of boundary layer physics is that they are distinct, and hence governed by their own mixing rules. An established basis for these rules is similarity theory, where the fundamental premise is that in a turbulent surface layer, dependent variables only depend on the distance from the surface and the forcing. Thus, Stokes drift is considered a forcing and the dot products in (10) and (11) accounted for. Hence, the mixing rules can now include the Stokes similarity functions in the surface layer and a challenge going forward is to discover if these functions play a role deeper in the boundary layer. An implication of the Wyngaard (1982) view and similarity theory is that in a boundary layer turbulent correlations such as the vertical fluxes $\left\langle w^{\prime} u^{\prime}\right\rangle,\left\langle w^{\prime} v^{\prime}\right\rangle$, and $\left\langle w^{\prime} \theta^{\prime}\right\rangle$ should depend on the forcing and hence on the production of TKE, rather than on turbulent quantities such as TKE itself, or its components, such as $\left\langle w^{2}\right\rangle$, or its dissipation. As a first step to a practical application, the turbulent velocity scales $w_{m}$ and $w_{s}$ have been parameterized in section 5 , in terms of the surface forcing plus the surface layer Stokes drift.

Adapting Monin-Obukhov similarity theory to the surface layer of the Southern Ocean LES confirms empirical results from the atmosphere, namely, $\kappa=0.40$, $\varepsilon=0.1$. It is necessary to determine the near surface limit of this layer $d_{o}$ empirically for each forcing regime. With a judicious choice of independent variables, the extended dimensional analysis of section $4 \mathrm{~b}$ 
remains compatible with this and other No-Stokes empiricism. Misalignment of the stress and shear vectors is accommodated by integrating the TKE production terms across the surface layer; given the Stokes drift profile as a forcing. The additional dimensionless group becomes the Stokes parameter $\xi$, the ratio of Stokes to total surface layer production of TKE. This ratio has a similar basis as the stability parameter $d / L$, because the Monin-Obukhov depth $L$ is where the rate of shear production equals buoyant production, or suppression (Wyngaard 2010).

The unequal and variable values of $P_{U}, P_{S}$, and $P_{B}$ are key to formulating the Stokes similarity functions $\chi_{m}(\varepsilon)$ and $\chi_{s}(\varepsilon)$ of the Stokes parameter (Fig. 7). These functions are the culmination of a multistep analysis: $\zeta$ from (20) rather than from (1), depth averaging, and the Stokes similarity functions (24) and (25) of $\xi$. Each step reduces the spread, as quantified by comparable standard deviations. The overall reductions are from 0.33 to 0.036 for $\chi_{m}$, and from 0.15 to 0.032 for $\chi_{s}$. Thus, the spread becomes significantly smaller than the No-Stokes standard deviations of 0.12 (Fig. 5c) and 0.20 (Fig. 5d), which offers the welcome prospect that ocean boundary layers may be better modeled with Stokes forcing than without.

The frequency of occurrence distribution of Belcher et al. (2012), is mentioned in section 3. It is divided into regimes dominated by production due to Eulerian shear, to Stokes shear, and to buoyancy, that they term wind, Langmuir, and convection, respectively. These regimes are based LES calculations of TKE dissipation at $\sigma=0.5$, and the implicit assumption that this dissipation is representative of mixing in the unstable boundary layer. They find that Stokes effects are dominant (account for more than $90 \%$ of this dissipation) in the regime ( $\mathrm{La}<0.3 ; h / L_{L}<0.08$ ), which would not be unusual, whereas the Eulerian shear would seldom be important. To reach a similar conclusion using the average TKE production over the surface layer would require $P_{U}$ from (10), $P_{S}$ from (11), and $P_{B}$ from (12) to be approximately equal, but they are not. Disregarding variability, but taking $P_{B}=0.09, P_{S}=0.5$ (Fig. 8), and $P_{U}=2.5$ (Fig. 9) shifts the Stokes (Langmuir) regime to (La $\left.<0.13 ; h / L_{L}<0.4\right)$, which would be a rare occurrence. Although this shift leaves more of a role for convection and Eulerian shear, the Stokes shear production is still the biggest contributor (about 60\%) near the peak of the distribution. This result is consistent with Fig. 7 where $\xi$ is the Stokes contribution to the LES production of TKE in the surface layer, and falls between one-third and $85 \%$.

The parameterized $w_{m}^{\text {PAR }}$ and $w_{s}^{\text {PAR }}$ from the NoStokes cases (AprN and JunN) are highly correlated with LES equivalents (Fig. 10), and the positive biases suggest that the viscosity and diffusivity in (13) and (14) may not increase quite so rapidly with depth. With Stokes forcing these velocity scales are enhanced by unequal $\chi_{m}^{-1}>\chi_{s}^{-1}>1$ (Fig. 7). With sufficient Stokes forcing, $\chi_{m}$ becomes smaller than $\chi_{s}$ by enough to make Pr exceed unity. This behavior implies that Langmuir turbulence is more effective at eroding momentum gradients, than buoyancy gradients, but it is not captured by all models. For example, Chor et al. (2018) always has $\operatorname{Pr}=1$. Some parameterizations formulate turbulent velocity scales based on (30), with various formulations of the enhancement factor. However, with $\operatorname{Pr}<1$ in the surface layer they cannot represent both momentum and buoyancy features of the LES, even when buoyancy effects such as (32) are included. This problem is evident in Fig. 11, where $w_{m}^{\mathrm{MS}}$ and $w_{s}^{\text {MS }}$ are enhanced by the same factor and therefore only differ in unstable conditions where $\phi_{m}>\phi_{s}$ means $\operatorname{Pr}<1$. The same holds for $w_{m}^{\mathrm{SS}}$ and $w_{s}^{\mathrm{SS}}$ (Smyth et al. 2002), except the factor is different. The implementation of such a scheme by Li et al. (2016) attempts to account for wind-wave misalignment following Roekel et al. (2012), but Pr is unchanged because the same correction is applied to both velocity scales.

From a practical modeling point of view, a Stokes drift profile requirement is a complication. The offline procedure of section 3 requires considerable expertise that is not commonly available. Unfortunately, the LES solutions are not well represented by functions of Langmuir number alone, such as (30). However, a conclusion is that the Stokes drift profile does provide sufficient information about the Stokes forcing to be an effective independent variable in the dimensional analysis extending similarity theory. A simple approximation such as the bulk Stokes drift (BSD) of section 6 is an attractive alternative. However, the biases and scatter may be unacceptable for some applications, especially when the Stokes forcing is less prominent $(\xi<0.30)$ than in the Southern Ocean. It is becoming more common to couple a wave prediction model (e.g., WAVEWATCH III) to global models such as CESM2 (Li et al. 2016), but the required infrastructure is extensive and not readily available for all applications. Therefore, modeling surface wave effects globally and in all seasons remains a challenge. For example Li and Fox-Kemper (2017) show improvements in Southern Ocean mixed layer depths, especially in austral summer, but overall degradation between $40^{\circ} \mathrm{S}$ and $70^{\circ} \mathrm{N}$ in summer and between $50^{\circ} \mathrm{S}$ and $50^{\circ} \mathrm{N}$ in winter.

Acknowledgments. This work was made possible by support from the U.S. Department of Energy (DOE) 
under solicitation DE-FOA-0001036, Climate and Earth System Modeling: SciDAC and Climate Variability and Change, Grant SC-00126005, and the patience of Dorothy Koch is gratefully acknowledged, as are the contributions of other Principal Investigators; Todd Ringler, Gokhan Danabasoglu, and Matt Long, and constructive discussions with Dan Whitt. The National Center for Atmospheric Research (NCAR) is sponsored by the National Science Foundation under Cooperative Agreement 1852977 . LR was partially supported by the Office of Naval Research (N00014-16-1-2936). The four SOFS simulations utilized resources of the National Energy Research Scientific Computing Center, a DOE Office of Science User Facility supported by the Office of Science of the U.S. Department of Energy under Contract DE-AC02-05CH11231. The idealized simulations utilized high-performance computing on Yellowstone (ark:/85065/d7wd3xhc) provided by NCAR's Computational and Information Systems Laboratory. The simulations can be made available upon request to the corresponding author, in accordance with the data policies of DOE, NSF, and NCAR.

\section{REFERENCES}

Alves, J., M. Banner, and I. Young, 2003: Revisiting the PiersonMoskowitz asymptotic limits for fully developed wind waves. J. Phys. Oceanogr., 33, 1301-1323, https://doi.org/10.1175/ 1520-0485(2003)033<1301:RTPALF>2.0.CO;2.

Belcher, S., and Coauthors, 2012: A global perspective on mixing in the ocean surface boundary layer. J. Geophys. Res., 39, L18605, https://doi.org/10.1029/2012GL052932.

Berrisford, P., and Coauthors, 2011: The ERA-Interim Archive: Version 2.0. ERA Rep. Series 1, 23 pp., https://www.ecmwf.int/ en/elibrary/8174-era-interim-archive-version-20.

Carl, D., T. Tarbell, and H. A. Panofsky, 1973: Profiles of wind and temperature from towers over homogeneous terrain. J. Atmos. Sci., 30, 788-794, https://doi.org/10.1175/1520-0469(1973) $030<0788$ :POWATF $>2.0$. CO;2.

Chor, T., D. Yang, C. Meneveau, and M. Chameki, 2018: A turbulence velocity scale for predicting the fate of buoyant materials in the oceanic mixed layer. Geophys. Res. Lett., 45, 11 817-11 826, https://doi.org/10.1029/2018GL080296.

Craik, A., and S. Leibovich, 1976: A rational model for Langmuir circulations. J. Fluid Mech., 73, 401-426, https://doi.org/ 10.1017/S0022112076001420.

Danabasoglu, G., S. Bates, B. Briegleb, S. Jayne, M. Jochum, W. Large, S. Peacock, and S. Yeager, 2012: The CCSM4 ocean component. J. Climate, 25, 1361-1389, https://doi.org/10.1175/ JCLI-D-11-00091.1.

Downes, S., and Coauthors, 2014: An assessment of Southern Ocean water masses and sea ice during 1988-2007 in a suite of interannual CORE-II simulations. Ocean Modell., 94, 67-94, https://doi.org/10.1016/j.ocemod.2015.07.022.

DuVivier, A., W. Large, and R. Small, 2018: Argo observations of the deep mixing band in the southern ocean: A salinity modeling challenge. J. Geophys. Res. Oceans, 123, 7599-7617, https://doi.org/10.1029/2018JC014275.
Foken, T., 2006: 50 years of the Monin-Obukhov similarity theory. Bound.-Layer Meteor., 119, https://doi.org/10.1007/ s10546-006-9048-6.

Fox-Kemper, B., and Coauthors, 2011: Parameterization of mixed layer eddies. Part III: Implementation and impact in global ocean climate simulations. Ocean Modell., 39, 61-78, https:// doi.org/10.1016/j.ocemod.2010.09.002.

Frech, M., and L. Mahrt, 1995: A two-scale mixing formulation for the atmospheric boundary layer. Bound.-Layer Meteor., 73, 91-104, https://doi.org/10.1007/BF00708931.

Grant, A., and S. Belcher, 2009: Characteristics of Langmuir turbulence in the ocean mixed layer. J. Phys. Oceanogr., 39, 18711887, https://doi.org/10.1175/2009JPO4119.1.

Hamlington, P. E., L. P. van Roekel, B. Fox-Kemper, K. Julien, and G. Chini, 2014: Langmuir-submesoscale interactions: Descriptive analysis of multiscale frontal spindown simulations. J. Phys. Oceanogr., 44, 2249-2272, https://doi.org/10.1175/ JPO-D-13-0139.1.

Harcourt, R., and E. D'Asaro, 2008: Large-eddy simulation of Langmuir turbulence in pure wind seas.J. Phys. Oceanogr., 38, 1542-1562, https://doi.org/10.1175/2007JPO3842.1.

Högström, U., 1988: Non-dimensional wind and temperature profiles in the atmospheric surface layer. Bound.-Layer Meteor., 42, 55-78, https://doi.org/10.1007/BF00119875.

Khatiwala, S., F. Primeau, and T. Hall, 2009: Reconstruction of the history of anthropogenic $\mathrm{CO}_{2}$ concentrations in the ocean. Nature, 462, 346-349, https://doi.org/10.1038/nature08526.

Kouketsu, S., and Coauthors, 2011: Deep ocean heat content changes estimated from observation and reanalysis product and their influence on sea level change. J. Geophys. Res., 116, C03012, https://doi.org/10.1029/2010JC006464.

Kukulka, T., A. Plueddeman, and P. Sullivan, 2013: Inhibited upper ocean restratification in nonequilibrium swell conditions. Geophys. Res. Lett., 40, 3672-3676, https://doi.org/10.1002/ grl.50708.

Large, W., J. McWilliams, and S. Doney, 1994: Oceanic vertical mixing: A review and a model with a nonlocal boundary layer parameterization. Rev. Geophys., 32, 363-403, https://doi.org/ 10.1029/94RG01872.

Li, Q., and B. Fox-Kemper, 2017: Assessing the effects of Langmuir turbulence on the entrainment buoyancy flux in the ocean surface boundary layer. J. Phys. Oceanogr., 47, 2863-2886, https://doi.org/10.1175/JPO-D-17-0085.1.

— - , A. Craig, G. Danabasoglu, W. Large, and M. Vertenstein, 2016: Langmuir mixing effects on global climate: WAVEWATCH III in CESM. Ocean Modell., 103, 145160, https://doi.org/10.1016/j.ocemod.2015.07.020.

McWilliams, J. C., 2016: Submesoscale currents in the ocean. Proc. Roy. Soc. London, 51A, 999-1022, https://doi.org/10.1098/ rspa.2016.0117.

— , and P. Sullivan, 2000: Vertical mixing by Langmuir circulations. Spill Sci. Technol. Bull., 6, 225-237, https://doi.org/ 10.1016/S1353-2561(01)00041-X.

— — - and C.-H. Moeng, 1997: Langmuir turbulence in the ocean. J. Fluid Mech., 334, 1-30, https://doi.org/10.1017/ S0022112096004375.

Mellor, G., and T. Yamada, 1982: Development of a turbulence closure model for geophysical fluid problems. Rev. Geophys., 20, 851-875, https://doi.org/10.1029/RG020i004p00851.

Moeng, C.-H., 1984: A large-eddy simulation model for the study of planetary boundary-layer turbulence. J. Atmos. Sci., $\mathbf{4 1}$, 2052-2062, https://doi.org/10.1175/1520-0469(1984)041<2052: ALESMF $>2.0 . \mathrm{CO} ; 2$. 
and P. Sullivan, 1994: A comparison of shear and buoyancy driven planetary-boundary-layer flows. J. Atmos. Sci., 51, 999-1022, https://doi.org/10.1175/1520-0469(1994)051<0999: $\mathrm{ACOSAB}>2.0 . \mathrm{CO} ; 2$

Monin, A., and A. Obukhov, 1954: Basic laws of turbulent mixing in the surface layer of the atmosphere. Tr. Geofiz. Inst., Akad. Nauk SSSR, 24, 163-187.

Purkey, S., and G. Johnson, 2010: Warming of global abyssal and deep southern ocean waters between the 1990s and 2000s: Contributions to global heat and sea level rise budgets. J. Climate, 23, 6336-6351, https://doi.org/10.1175/ 2010JCLI3682.1.

Roekel, L. V., B. Fox-Kemper, P. Sullivan, P. Hamlington, and S. Haney, 2012: The form and orientation of Langmuir cells for misaligned winds and waves. J. Geophys. Res., 117, C05001, https://doi.org/10.1029/2011JC007516.

Romero, L., and W. Melville, 2010a: Airborne observations of fetchlimited waves in the Gulf of Tehuantepec. J. Phys. Oceanogr., 40, 441-465, https://doi.org/10.1175/2009JPO4127.1.

—, and W. K. Melville, 2010b: Numerical modeling of fetchlimited waves in the Gulf of Tehuantepec. J. Phys. Oceanogr., 40, 466-486, https://doi.org/10.1175/2009JPO4128.1.

Schultz, E., S. Josey, and R. Verein, 2012: First air-sea flux mooring measurements in the Southern Ocean. Geophys. Res. Lett., 39 L16606, https://doi.org/10.1029/2012GL052290.

Smyth, W., E. Skylingstad, G. Crawford, and H. Wijesekera, 2002 Nonlocal fluxes and stokes drift effects in the K-profile parameterization. Ocean Dyn., 52, 104-115, https://doi.org/ 10.1007/s10236-002-0012-9.

Snyder, R., F. Dobson, J. Elliott, and R. Long, 1981: Array measurements of atmospheric pressure fluctuations above surface gravity waves. J. Fluid Mech., 102, 1-59, https://doi.org/ 10.1017/S0022112081002528

Sullivan, P., and J. McWilliams, 2018: Frontogenesis and frontal arrest of a dense filament in the oceanic surface boundary layer. J. Fluid Mech., 837, 341-380, https://doi.org/10.1017/ jfm.2017.833.

- and E. G. Patton, 2011: The effect of mesh resolution on convective boundary layer statistics and structures generated by large-eddy simulation. J. Atmos. Sci., 68, 2395-2415, https:// doi.org/10.1175/JAS-D-10-05010.1.

J. McWilliams, and C. Moeng, 1994: A subgrid-scale model for large-eddy simulation of planetary boundary-layer flows. Bound.-Layer Meteor., 71, 247-276, https://doi.org/10.1007/ BF00713741.

T. Horst, D. Lenschow, C.-H. Moeng, and J. Weil, 2003: Structure of subfilter-scale fluxes in the atmospheric surface layer with application to large-eddy simulation modeling. J. Fluid Mech., 482, 101-139, https://doi.org/10.1017/S0022112003004099.

J. McWilliams, and W. Melville, 2004: The oceanic boundary layer driven by wave breaking with stochastic variability. Part 1: Direct numerical simulations. J. Fluid Mech., 507, 143-174, https://doi.org/10.1017/S0022112004008882.

,-- , and -2007 : Surface gravity wave effects in the oceanic boundary layer: Large-eddy simulation with vortex force and stochastic breakers. J. Fluid Mech., 593, 405-452, https://doi.org/10.1017/S002211200700897X

- L. Lomero, J. McWilliams, and W. Melville, 2012: Transient evolution of Langmuir turbulence in ocean boundary layers driven by hurricane winds and waves. J. Phys. Oceanogr., 42, 1959-1980, https://doi.org/10.1175/JPO-D-12-025.1.

Tolman, H. L., 2002: User manual and system documentation of WAVEWATCH-III version 2.22. Tech. rep., NOAA/ NWS/NCEP/MMAB Tech. Note 222, 133 pp., https:// polar.ncep.noaa.gov/mmab/papers/tn222/MMAB_222.pdf.

van Vledder, G. Ph., 2006: The WRT method for the computation of non-linear four-wave interactions in discrete spectral wave models. Coastal Eng., 53, 223-242, https://doi.org/10.1016/ j.coastaleng.2005.10.011.

Weijer, W., and Coauthors, 2012: The Southern Ocean and its climate in CCSM4. J. Climate, 25, 2652-2675, https://doi.org/ 10.1175/JCLI-D-11-00302.1.

Wyngaard, J., 1982: Lectures on the planetary boundary layer. Mesoscale Meteorology - Theories, Observations and Models, D. Lilly and T. Gal-Chen, Eds., NATO ASI Series C, D. Reidel, 603-650. 2010: Turbulence in the Atmosphere. Cambridge University Press, $406 \mathrm{pp}$ 